'Supporting information for:

\title{
Unraveling Structures Containing Highly Conjugated Pyrrolo[4,3,2- de]quinoline Cores that are Deficient in Diagnostic Proton NMR Signals
}

\author{
Erin P. McCauley, Gavin C. Smith, Phillip Crews*
}

Department of Chemistry \& Biochemistry, University of California Santa Cruz, Santa Cruz, CA 95064

*To whom correspondence should be addressed

Tel: (831) 459-2603 Email: pcrews@ucsc.edu

\section{SUPPORTING INFORMATION}




\section{Table of Contents}

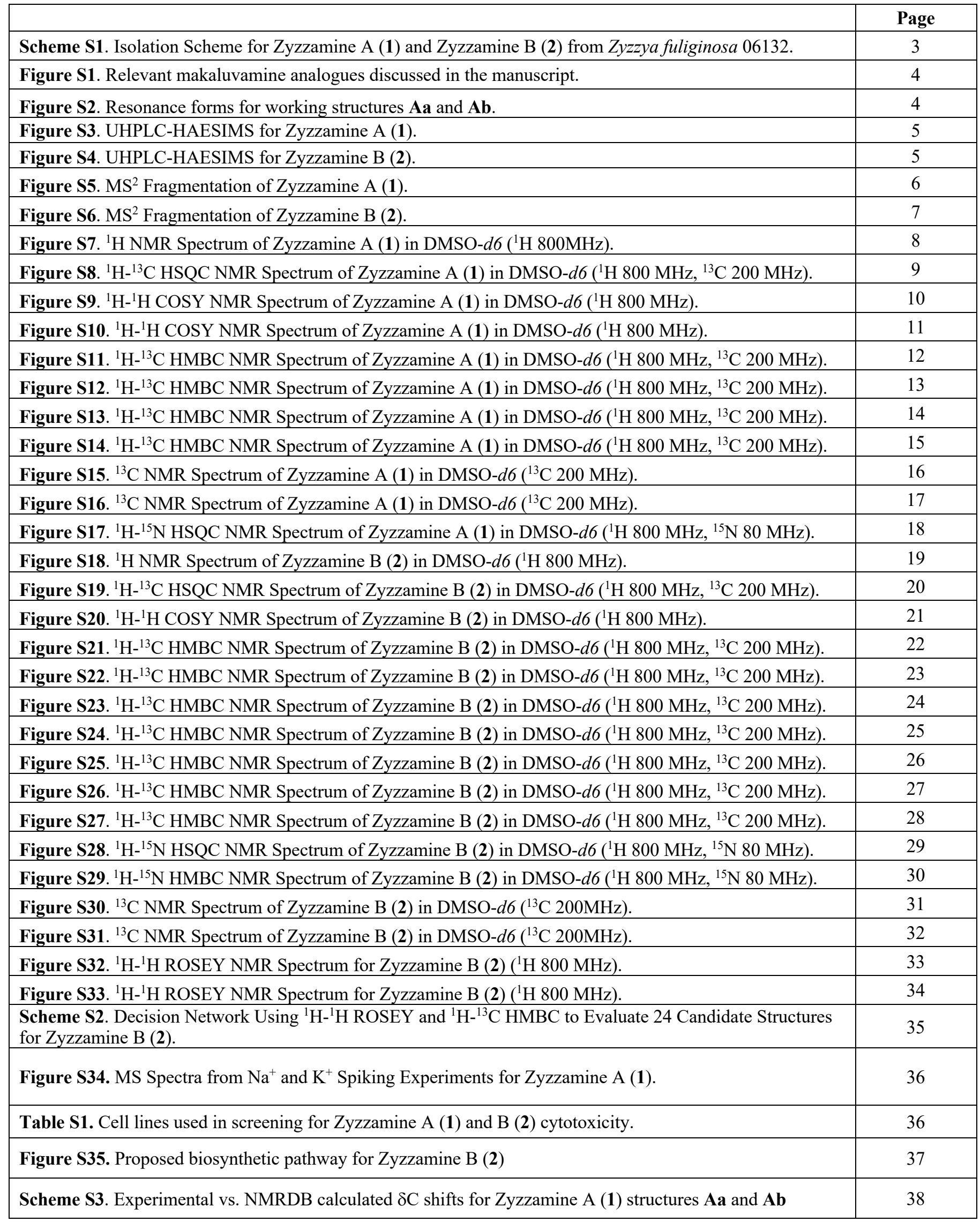




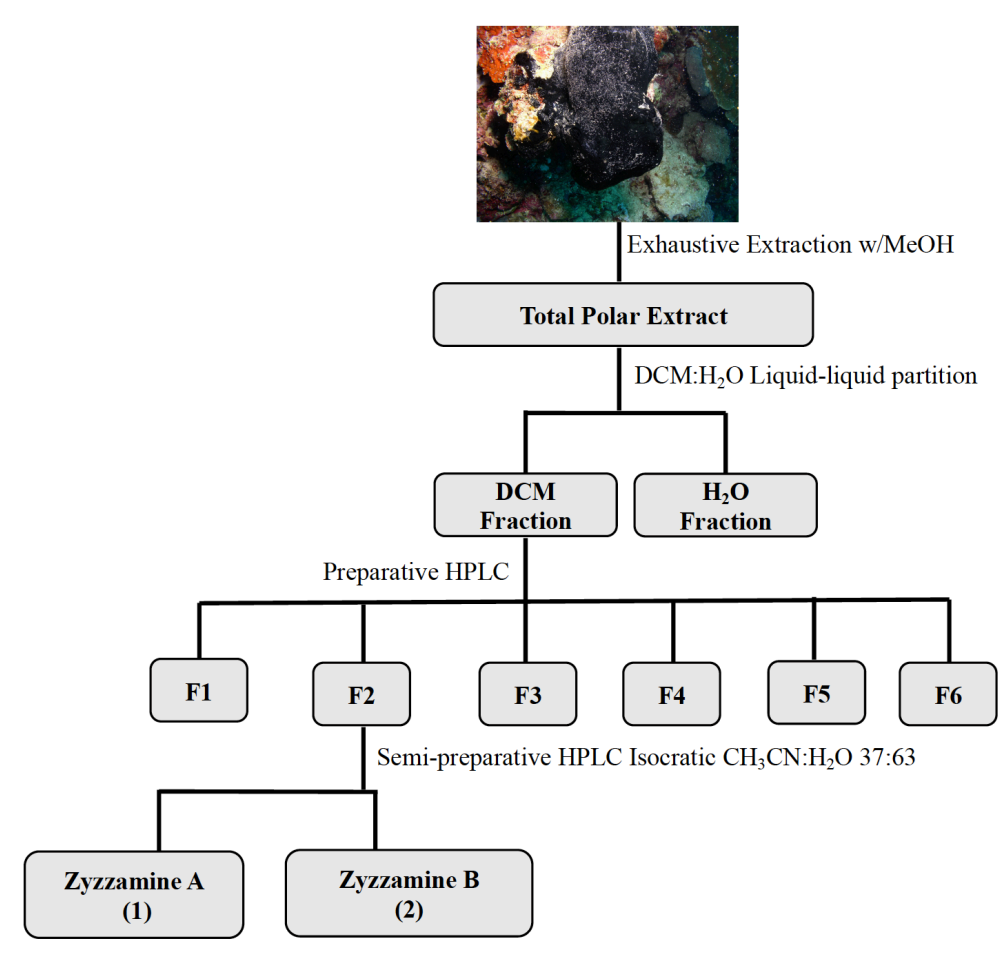

Scheme S1. Isolation Scheme for Zyzzamine A (1) and Zyzzamine B (2) from Zyzzya fuliginosa 06132. 
<smiles></smiles>

Makaluvamine $\mathrm{J}(9)$

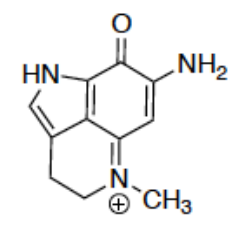

Makaluvamine C (10)

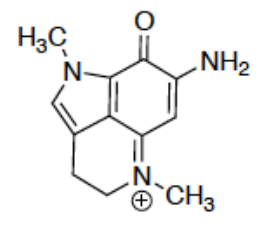

Makaluvamine H (11)

Figure S1. Relevant makaluvamine analogues discussed in the manuscript.
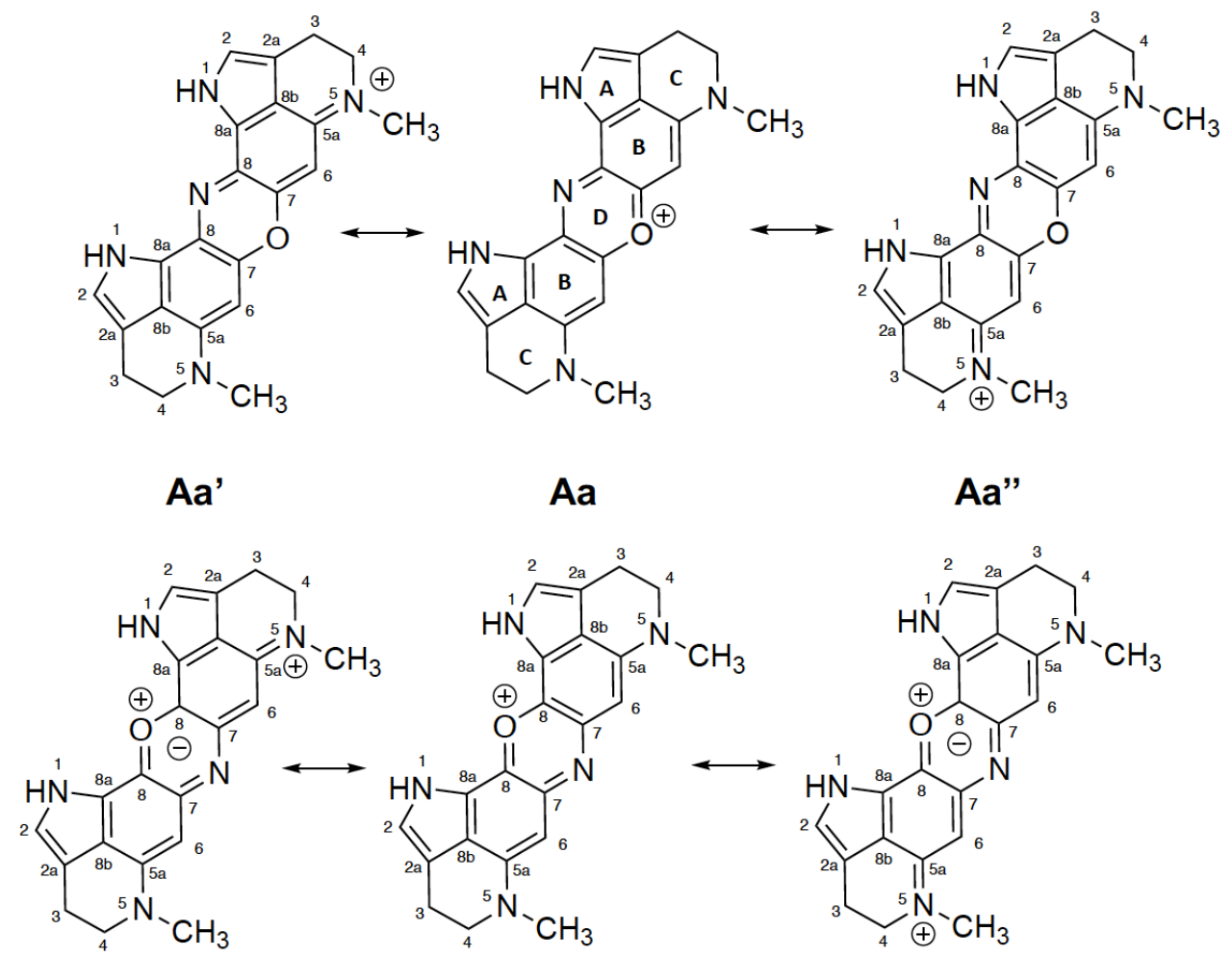

$A b^{\prime}$

Ab

Ab"

Figure S2. Resonance forms for working structures $\mathbf{A a}$ and $\mathbf{A b}$. 

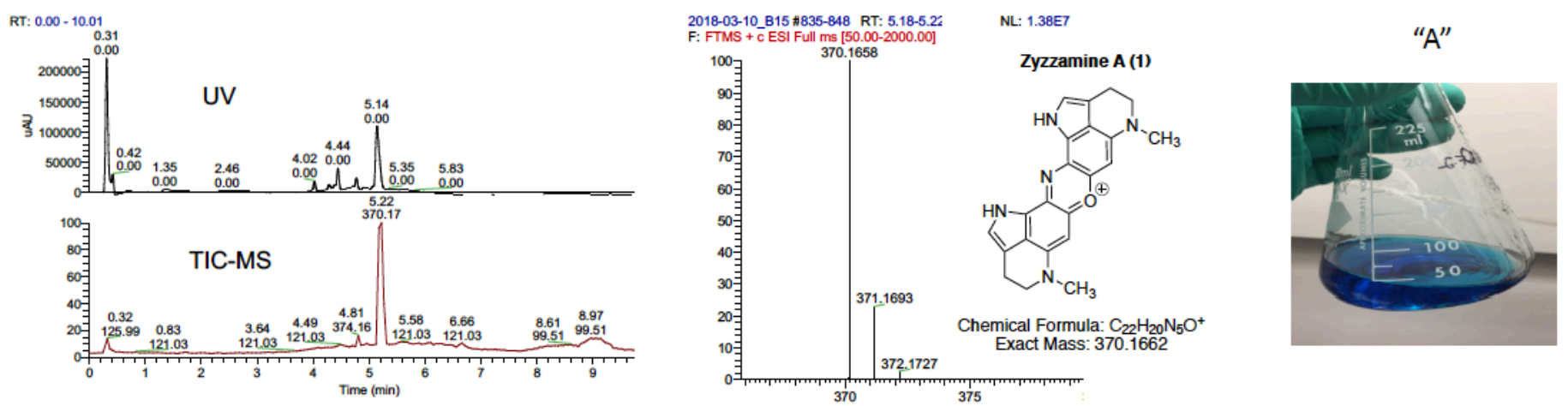

Figure S3. UHPLC-HAESIMS for Zyzzamine A (1).
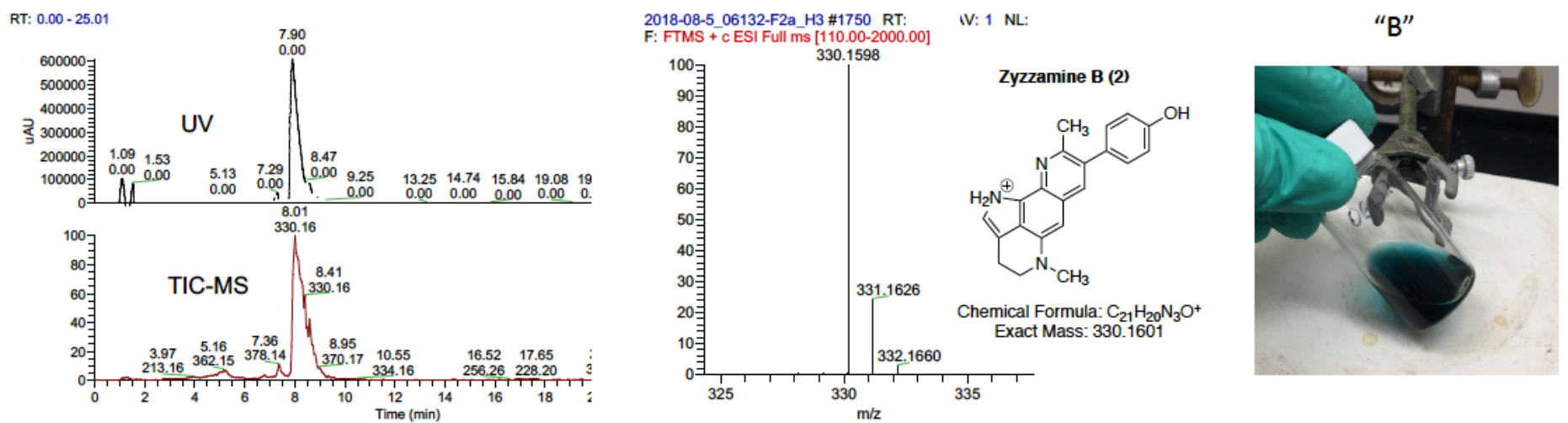

Figure S4. UHPLC-HAESIMS for Zyzzamine B (2). 


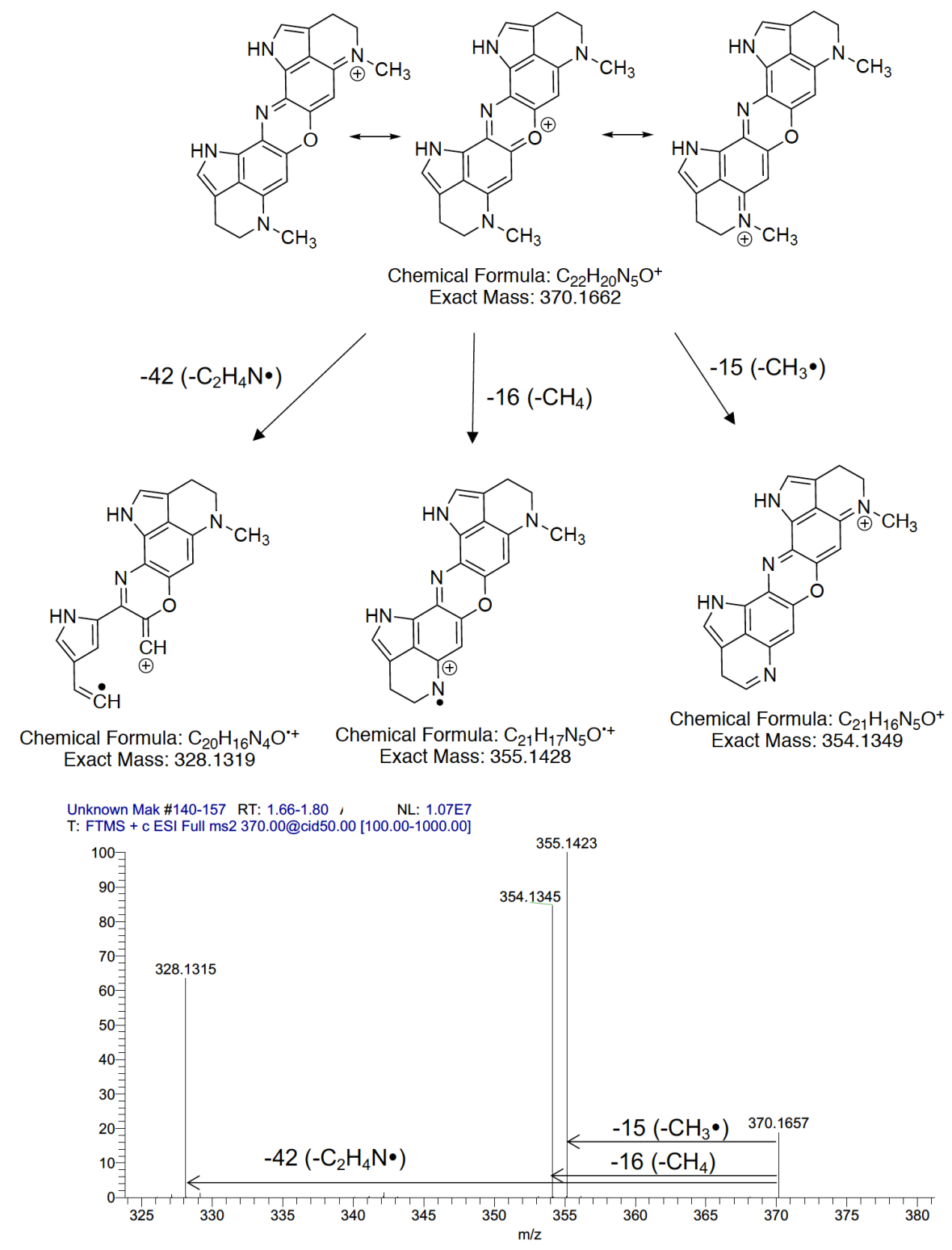

Figure S5. MS ${ }^{2}$ Fragmentation of Zyzzamine A (1). 


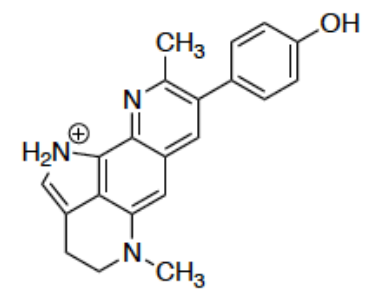

Chemical Formula: $\mathrm{C}_{21} \mathrm{H}_{20} \mathrm{~N}_{3} \mathrm{O}^{+}$

Exact Mass: 330.1601

06132 17 \#65-304 RT: 0.54-1.09 AV

$-15\left(-\mathrm{CH}_{3} \bullet\right)$

T: FTM $\mathrm{MS}+\mathrm{CESI}$ Full

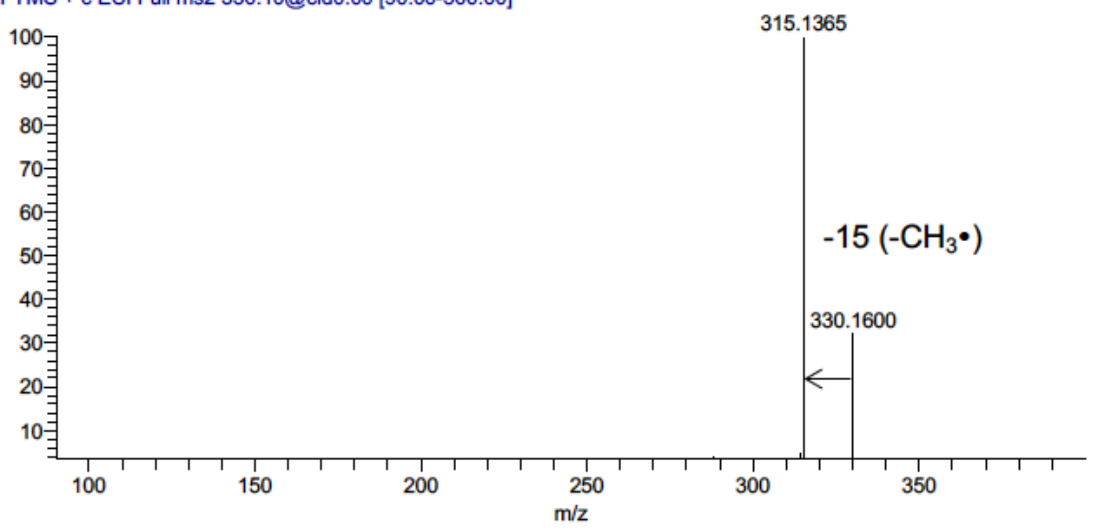

Figure S6. MS² Fragmentation of Zyzzamine B (2). 


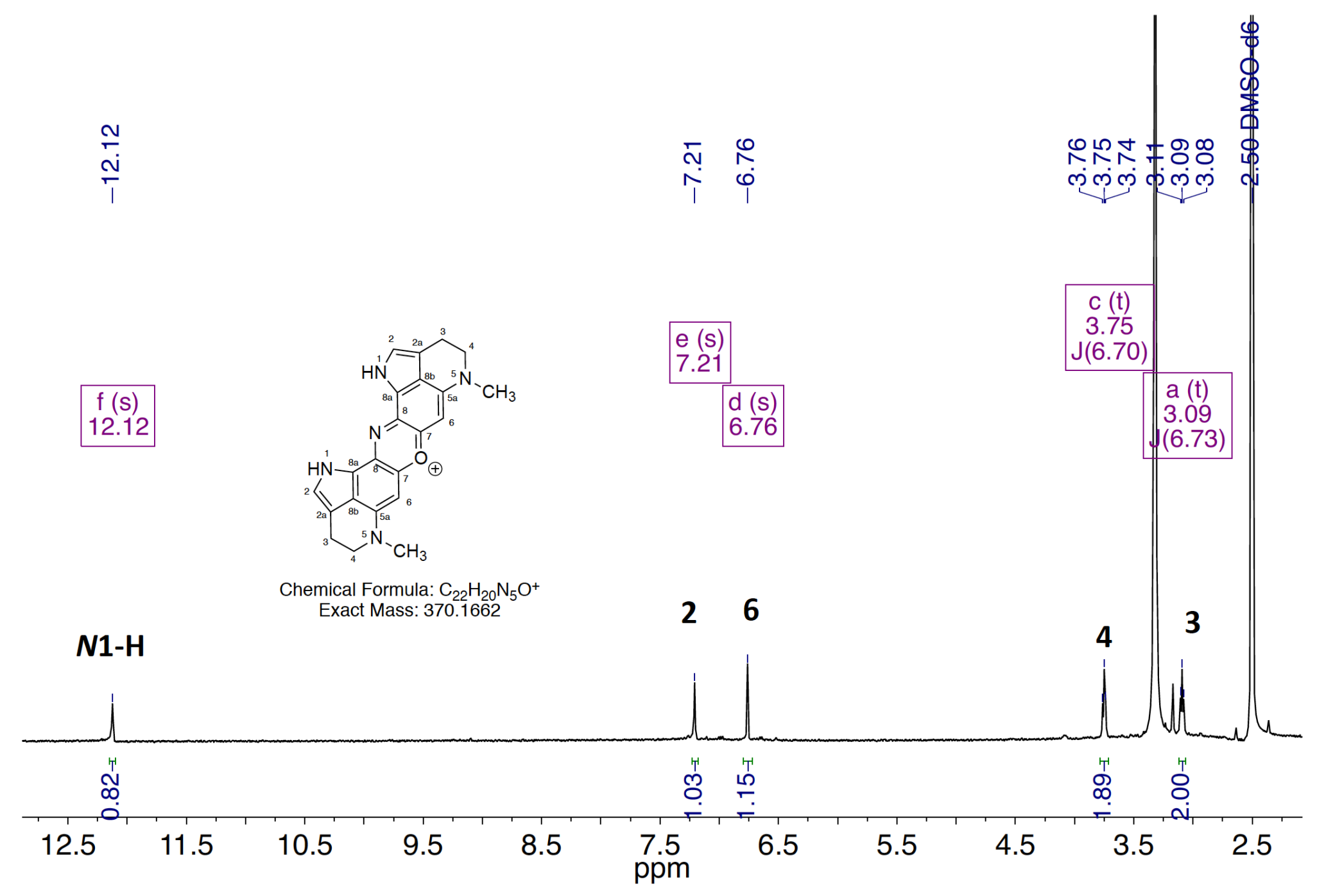

Figure S7. ${ }^{1} \mathrm{H}$ NMR Spectrum of Zyzzamine A (1) in DMSO-d6 $\left({ }^{1} \mathrm{H} 800 \mathrm{MHz}\right)$. 


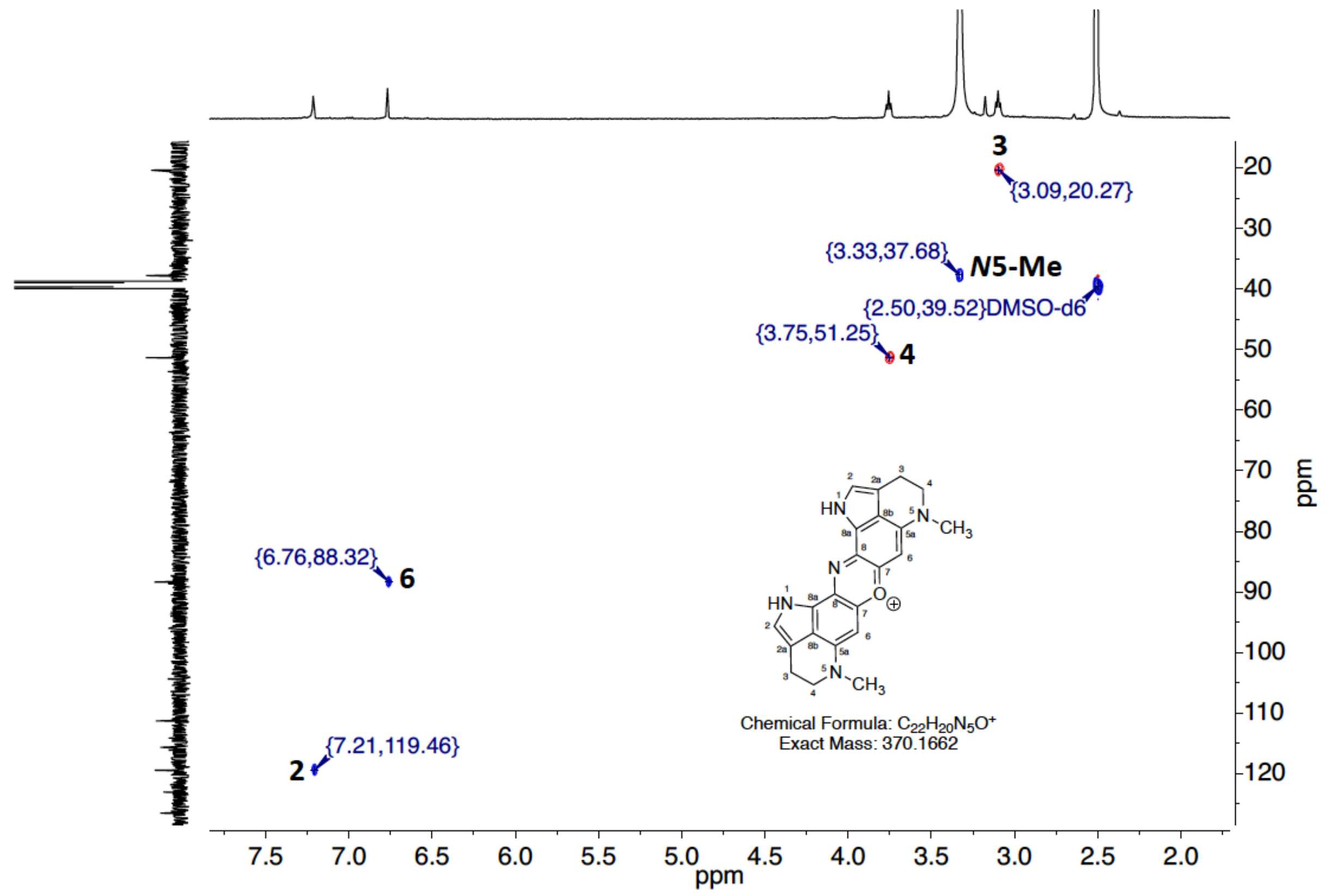

Figure S8. ${ }^{1} \mathrm{H}^{-13} \mathrm{C}$ HSQC NMR Spectrum of Zyzzamine A (1) in DMSO-d6 $\left({ }^{1} \mathrm{H} 800 \mathrm{MHz},{ }^{13} \mathrm{C} 200 \mathrm{MHz}\right)$. 


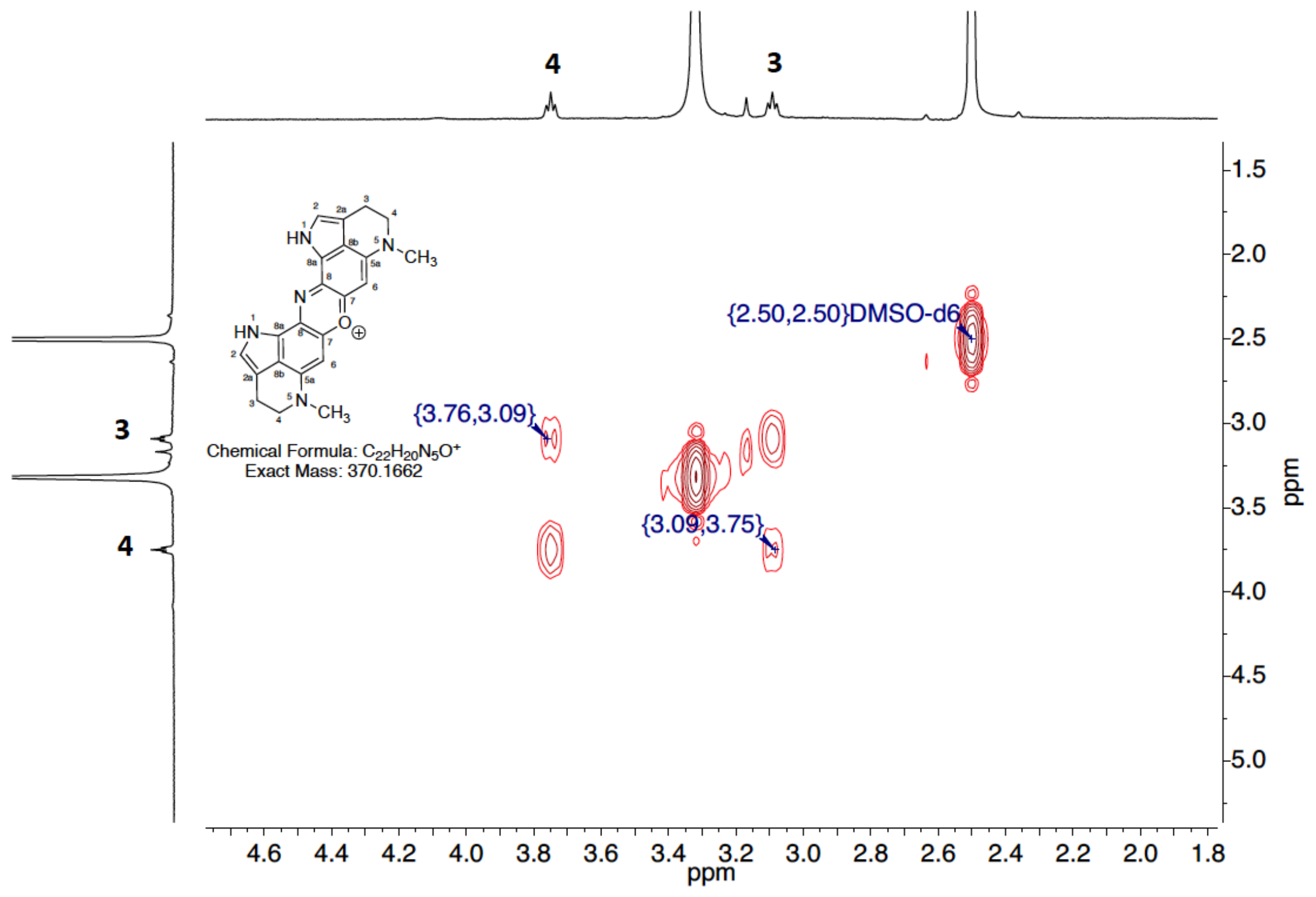

Figure S9. ${ }^{1} \mathrm{H}-{ }^{1} \mathrm{H}$ COSY NMR Spectrum of Zyzzamine A (1) in DMSO-d6 $\left({ }^{1} \mathrm{H} 800 \mathrm{MHz}\right)$. 




Figure S10. ${ }^{1} \mathrm{H}-{ }^{1} \mathrm{H}$ COSY NMR Spectrum of Zyzzamine A (1) in DMSO-d6 $\left({ }^{1} \mathrm{H} 800 \mathrm{MHz}\right)$. 




Figure S11. ${ }^{1} \mathrm{H}-{ }^{13} \mathrm{C}$ HMBC NMR Spectrum of Zyzzamine A (1) in DMSO-d6 $\left({ }^{1} \mathrm{H} 800 \mathrm{MHz},{ }^{13} \mathrm{C} 200 \mathrm{MHz}\right)$. 


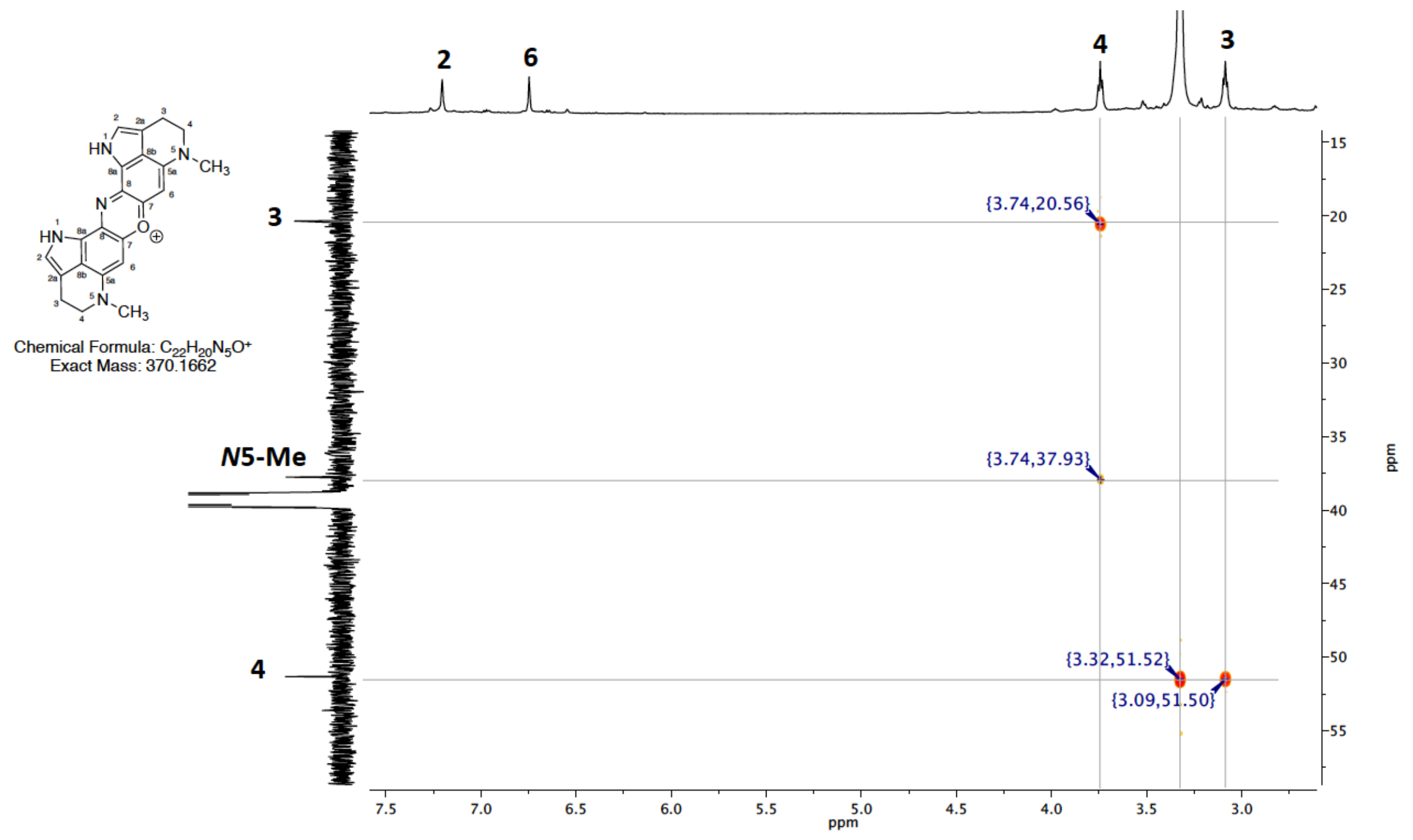

Figure S12. ${ }^{1} \mathrm{H}-{ }^{13} \mathrm{C}$ HMBC NMR Spectrum of Zyzzamine A (1) in DMSO-d6 $\left({ }^{1} \mathrm{H} 800 \mathrm{MHz},{ }^{13} \mathrm{C} 200 \mathrm{MHz}\right)$. 


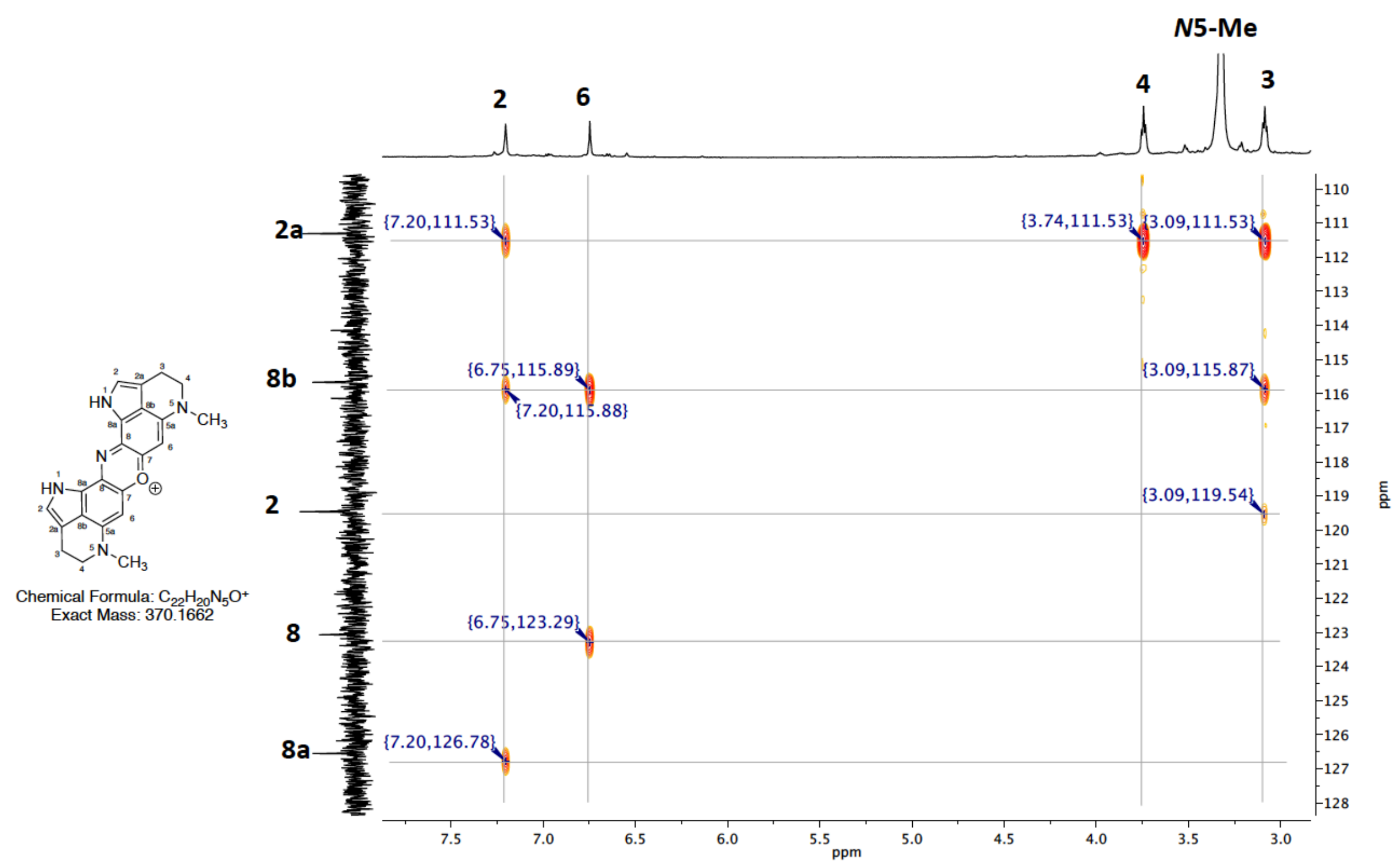

Figure S13. ${ }^{1} \mathrm{H}-{ }^{13} \mathrm{C}$ HMBC NMR Spectrum of Zyzzamine A (1) in DMSO-d6 $\left({ }^{1} \mathrm{H} 800 \mathrm{MHz},{ }^{13} \mathrm{C} 200 \mathrm{MHz}\right)$. 


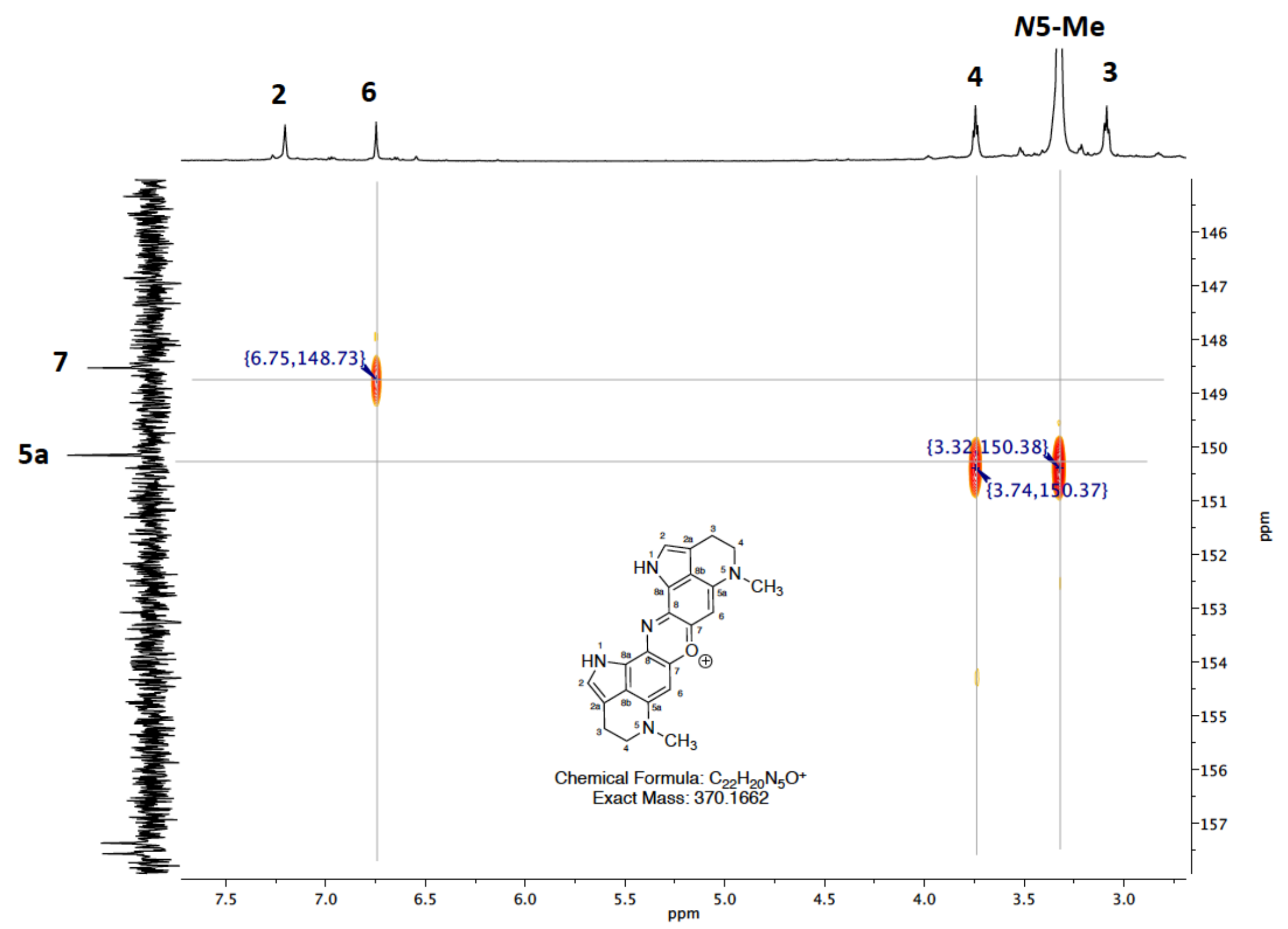

Figure S14. ${ }^{1} \mathrm{H}-{ }^{13} \mathrm{C}$ HMBC NMR Spectrum of Zyzzamine A (1) in DMSO-d6 $\left({ }^{1} \mathrm{H} 800 \mathrm{MHz},{ }^{13} \mathrm{C} 200 \mathrm{MHz}\right)$. 




Chemical Formula: $\mathrm{C}_{22} \mathrm{H}_{20} \mathrm{~N}_{5} \mathrm{O}^{+}$ Exact Mass: 370.1662
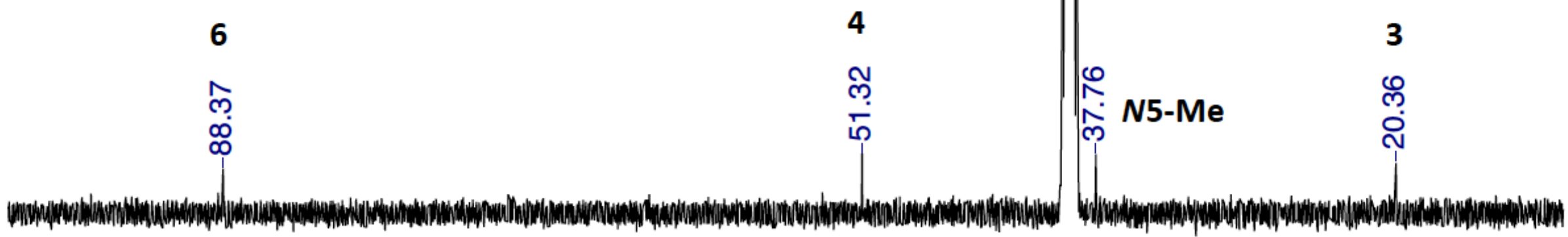

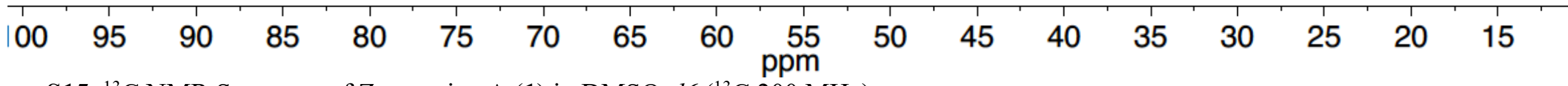

Figure S15. ${ }^{13} \mathrm{C}$ NMR Spectrum of Zyzzamine A (1) in DMSO-d6 $\left({ }^{13} \mathrm{C} 200 \mathrm{MHz}\right)$. 


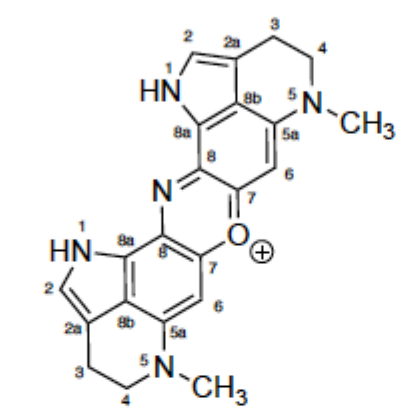

Chemical Formula: $\mathrm{C}_{22} \mathrm{H}_{20} \mathrm{~N}_{5} \mathrm{O}^{+}$ Exact Mass: 370.1662

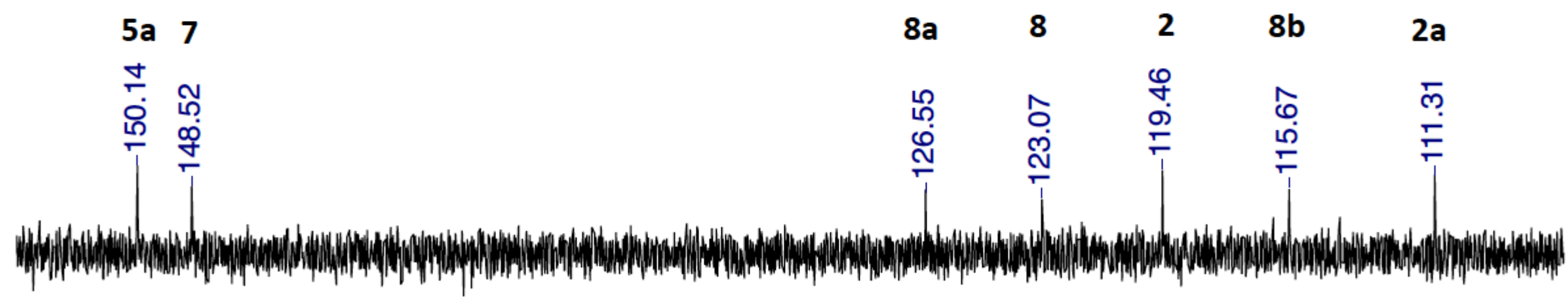

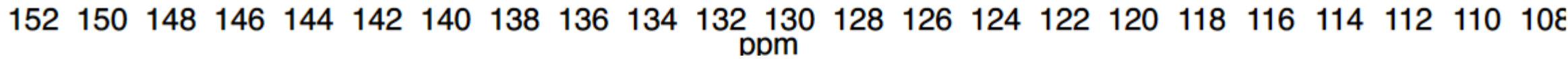

Figure S16. ${ }^{13} \mathrm{C}$ NMR Spectrum of Zyzzamine A (1) in DMSO-d6 $\left({ }^{13} \mathrm{C} 200 \mathrm{MHz}\right)$. 


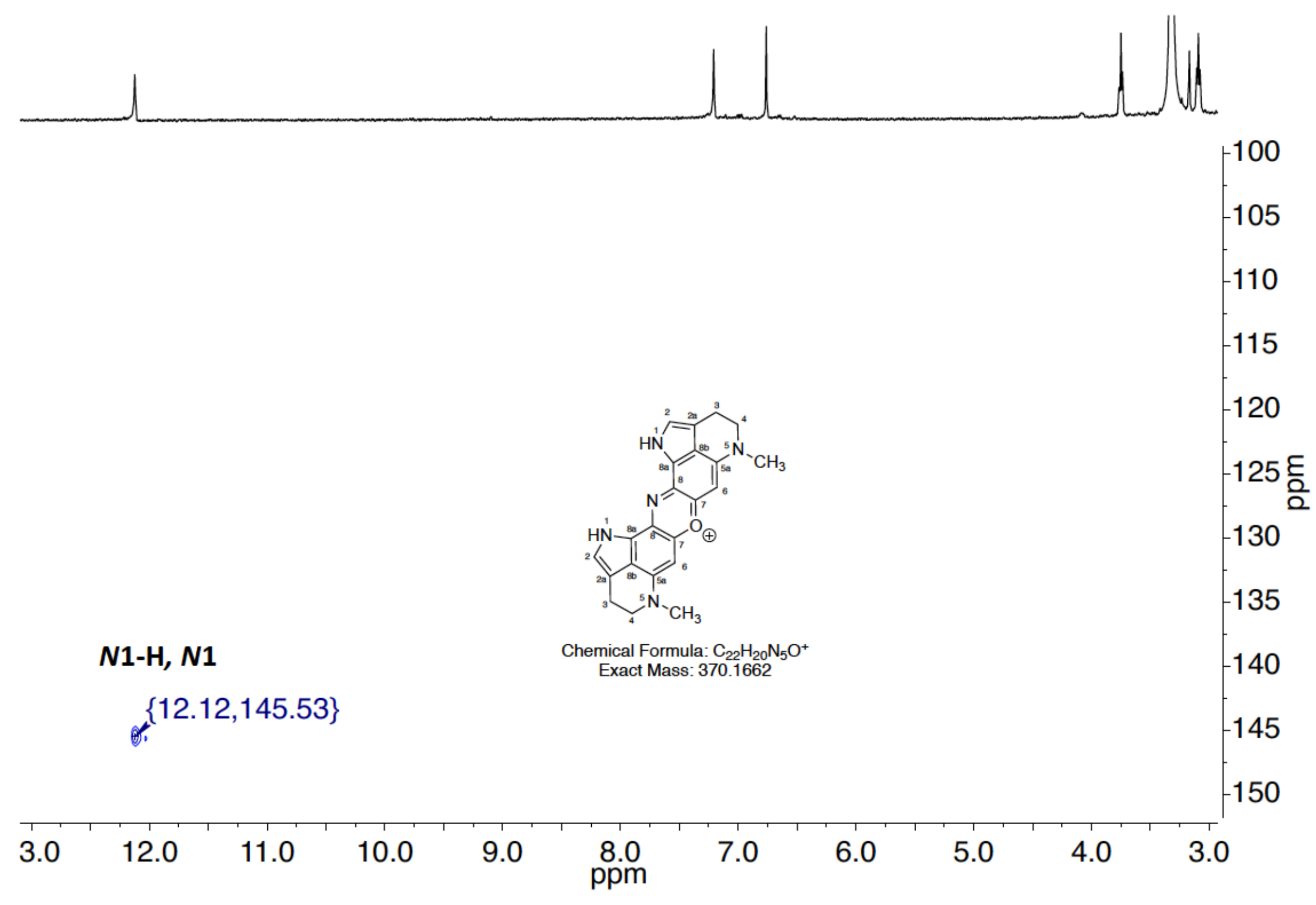

Figure S17. ${ }^{1} \mathrm{H}^{-15} \mathrm{~N}$ HSQC NMR Spectrum of Zyzzamine A (1) in DMSO-d6 $\left({ }^{1} \mathrm{H} 800 \mathrm{MHz},{ }^{15} \mathrm{~N} 80 \mathrm{MHz}\right)$. 

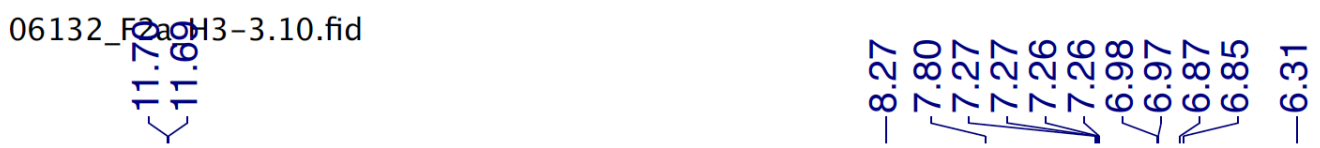


$\infty$ N N N

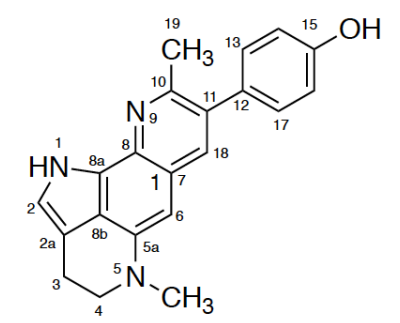

Chemical Formula: $\mathrm{C}_{21} \mathrm{H}_{19} \mathrm{~N}_{3} \mathrm{O}$

Exact Mass: 329.1528

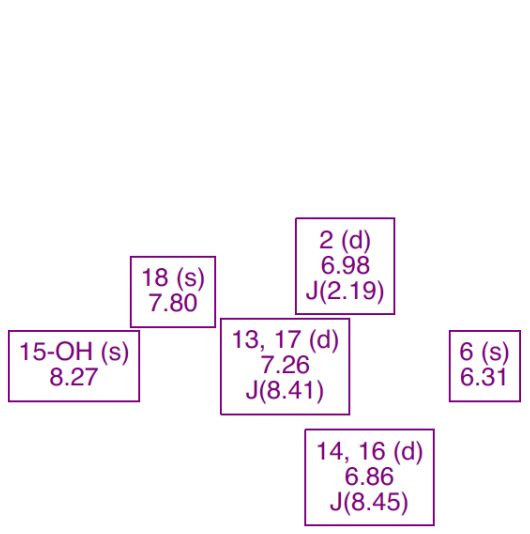

N5-Me

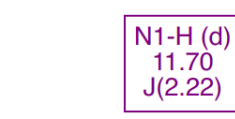

18

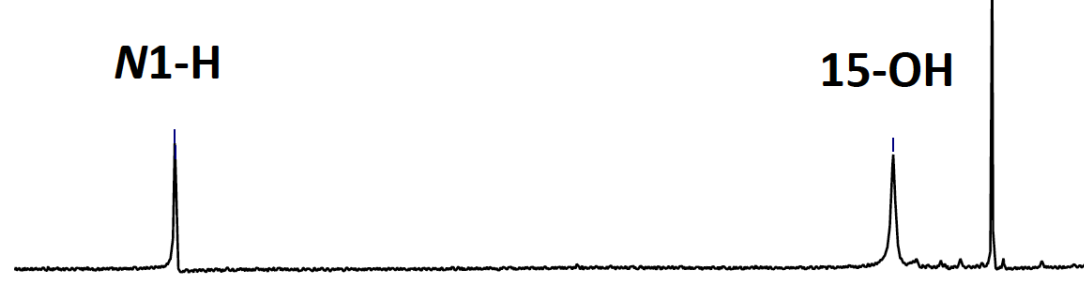

$1314 \quad 6$



Figure S18. ${ }^{1} \mathrm{H}$ NMR Spectrum of Zyzzamine B (2) in DMSO-d6 $\left({ }^{1} \mathrm{H} 800 \mathrm{MHz}\right)$. 


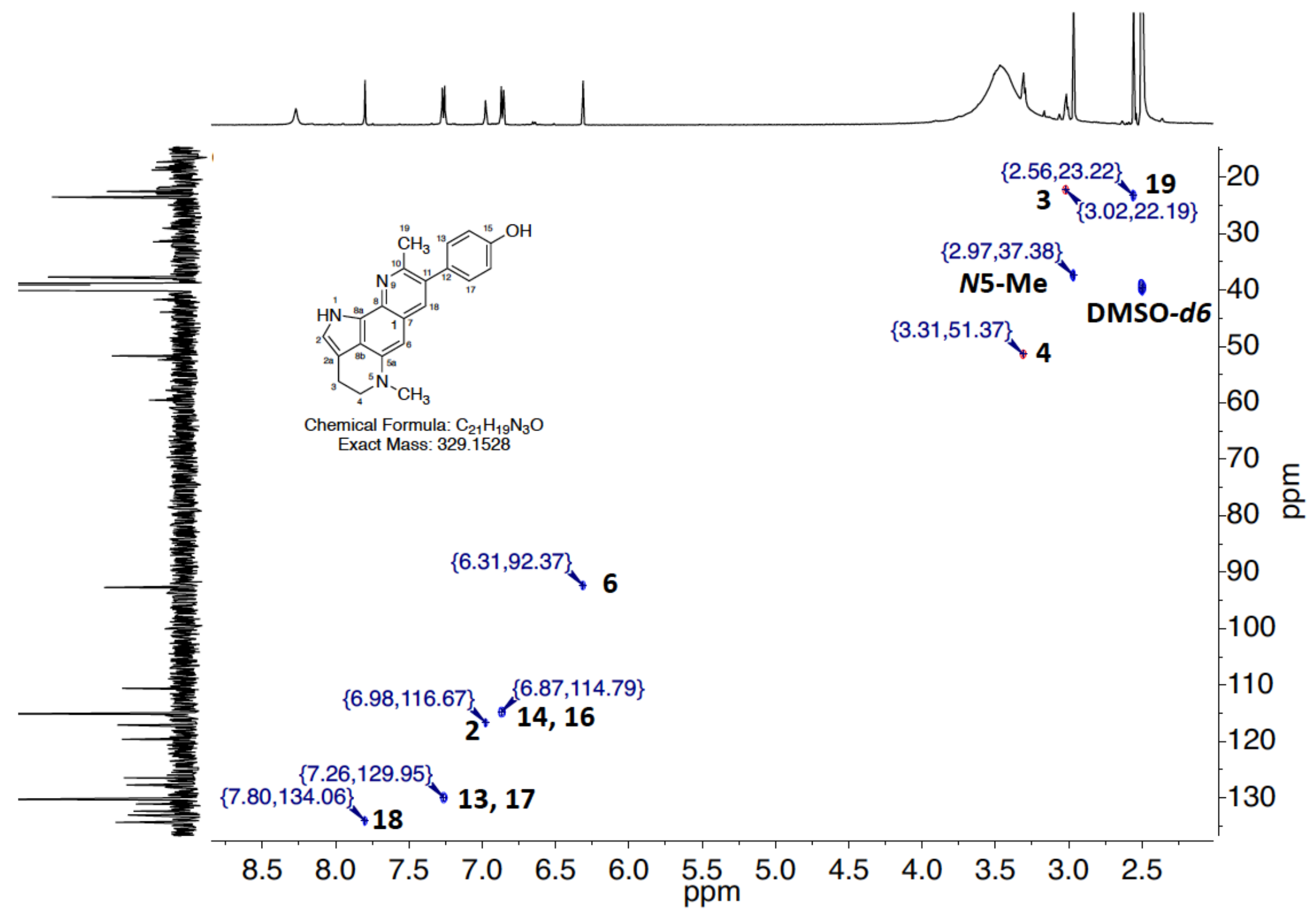

Figure S19. ${ }^{1} \mathrm{H}-{ }^{13} \mathrm{C}$ HSQC NMR Spectrum of Zyzzamine B (2) in DMSO-d6 $\left({ }^{1} \mathrm{H} 800 \mathrm{MHz},{ }^{13} \mathrm{C} 200 \mathrm{MHz}\right)$. 




Figure S20. ${ }^{1} \mathrm{H}-{ }^{1} \mathrm{H}$ COSY NMR Spectrum of Zyzzamine B (2) in DMSO-d6 $\left({ }^{1} \mathrm{H} 800 \mathrm{MHz}\right)$. 




Figure S21. ${ }^{1} \mathrm{H}-{ }^{13} \mathrm{C}$ HMBC NMR Spectrum of Zyzzamine B (2) in DMSO-d6 $\left({ }^{1} \mathrm{H} 800 \mathrm{MHz},{ }^{13} \mathrm{C} 200 \mathrm{MHz}\right)$. 


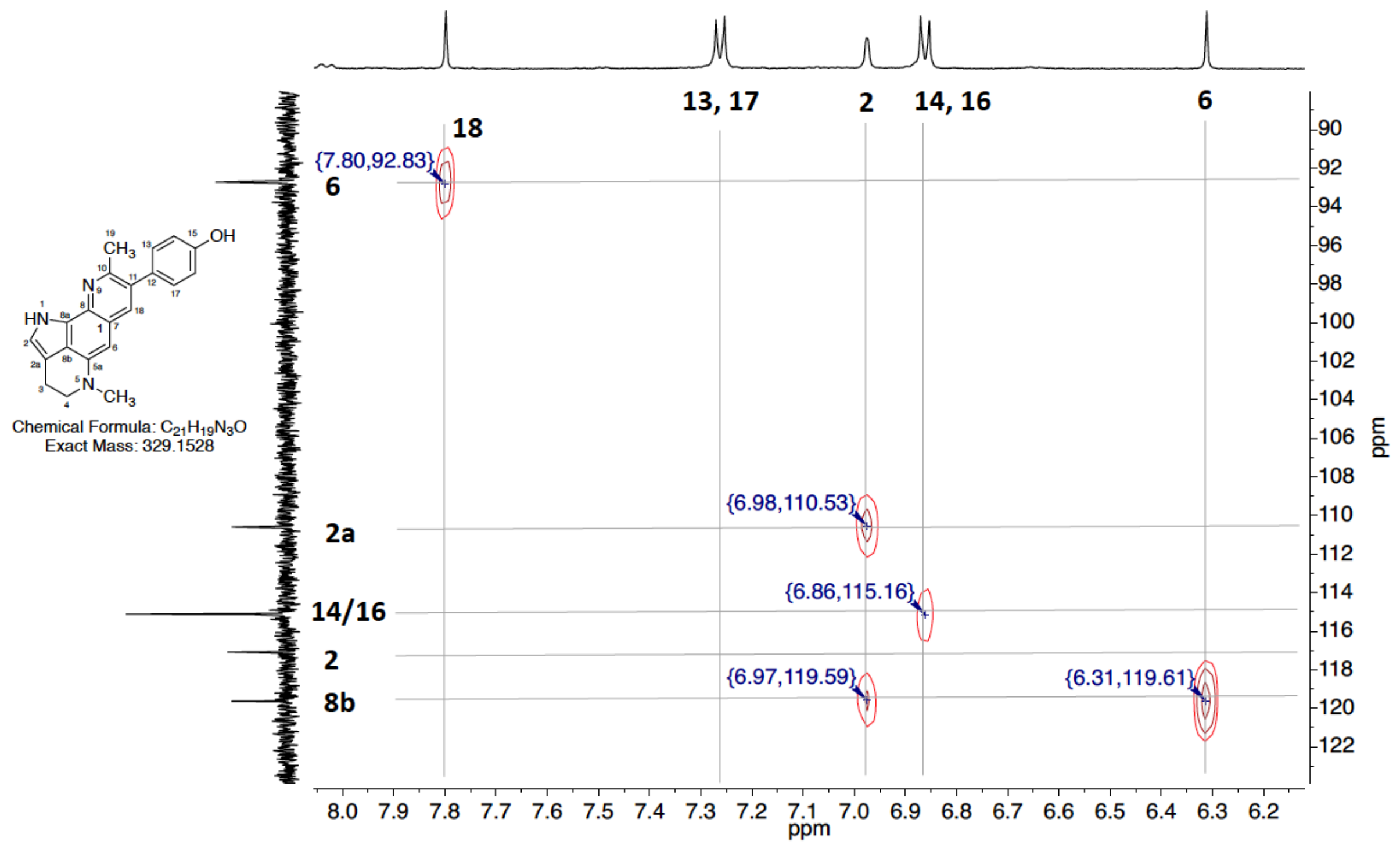

Figure S22. ${ }^{1} \mathrm{H}-{ }^{13} \mathrm{C}$ HMBC NMR Spectrum of Zyzzamine B (2) in DMSO-d6 $\left({ }^{1} \mathrm{H} 800 \mathrm{MHz},{ }^{13} \mathrm{C} 200 \mathrm{MHz}\right)$. 


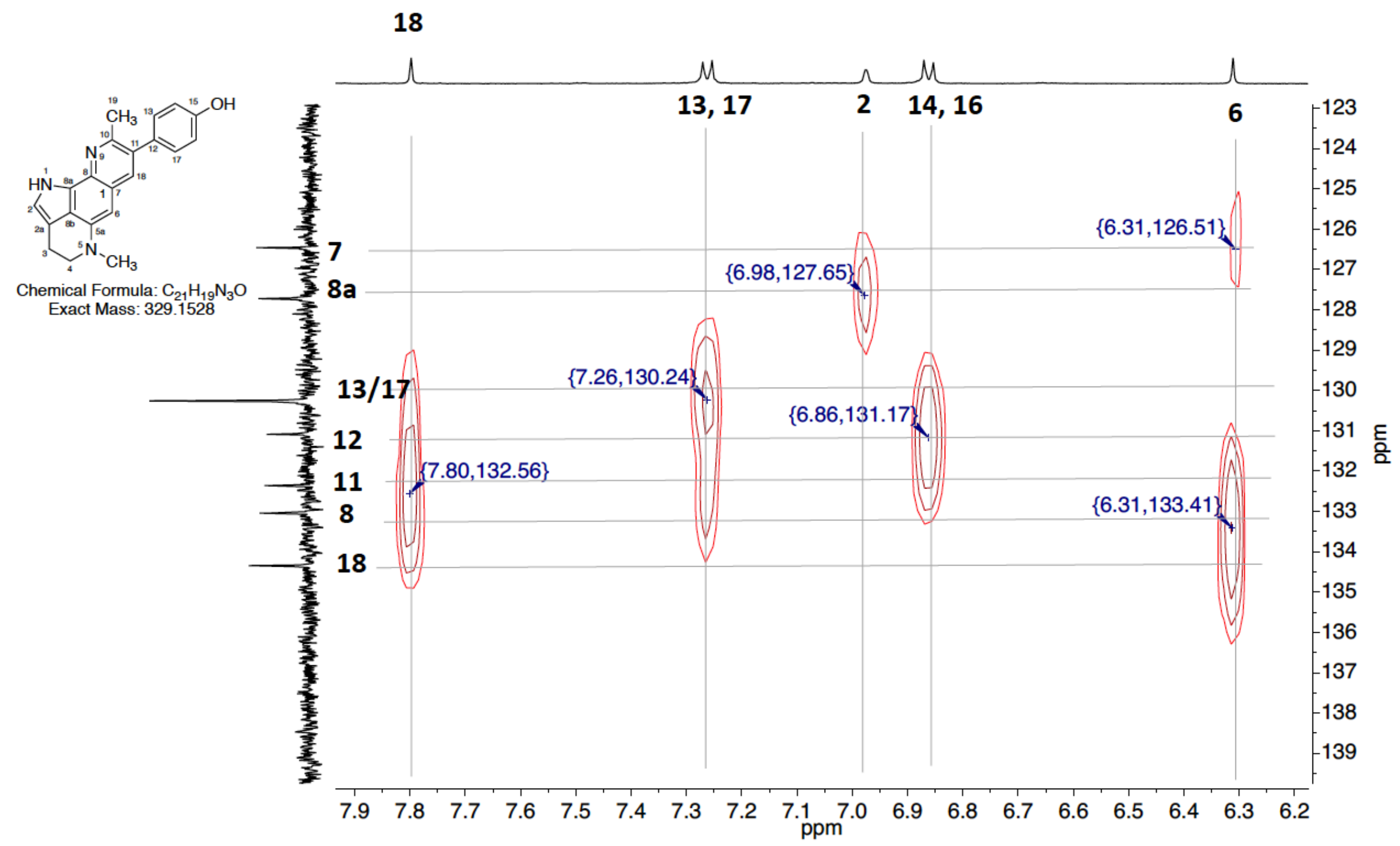

Figure S23. ${ }^{1} \mathrm{H}-{ }^{13} \mathrm{C}$ HMBC NMR Spectrum of Zyzzamine B (2) in DMSO-d6 $\left({ }^{1} \mathrm{H} 800 \mathrm{MHz},{ }^{13} \mathrm{C} 200 \mathrm{MHz}\right)$. 




Figure S24. ${ }^{1} \mathrm{H}-{ }^{13} \mathrm{C}$ HMBC NMR Spectrum of Zyzzamine B (2) in DMSO-d6 $\left({ }^{1} \mathrm{H} 800 \mathrm{MHz},{ }^{13} \mathrm{C} 200 \mathrm{MHz}\right)$. 


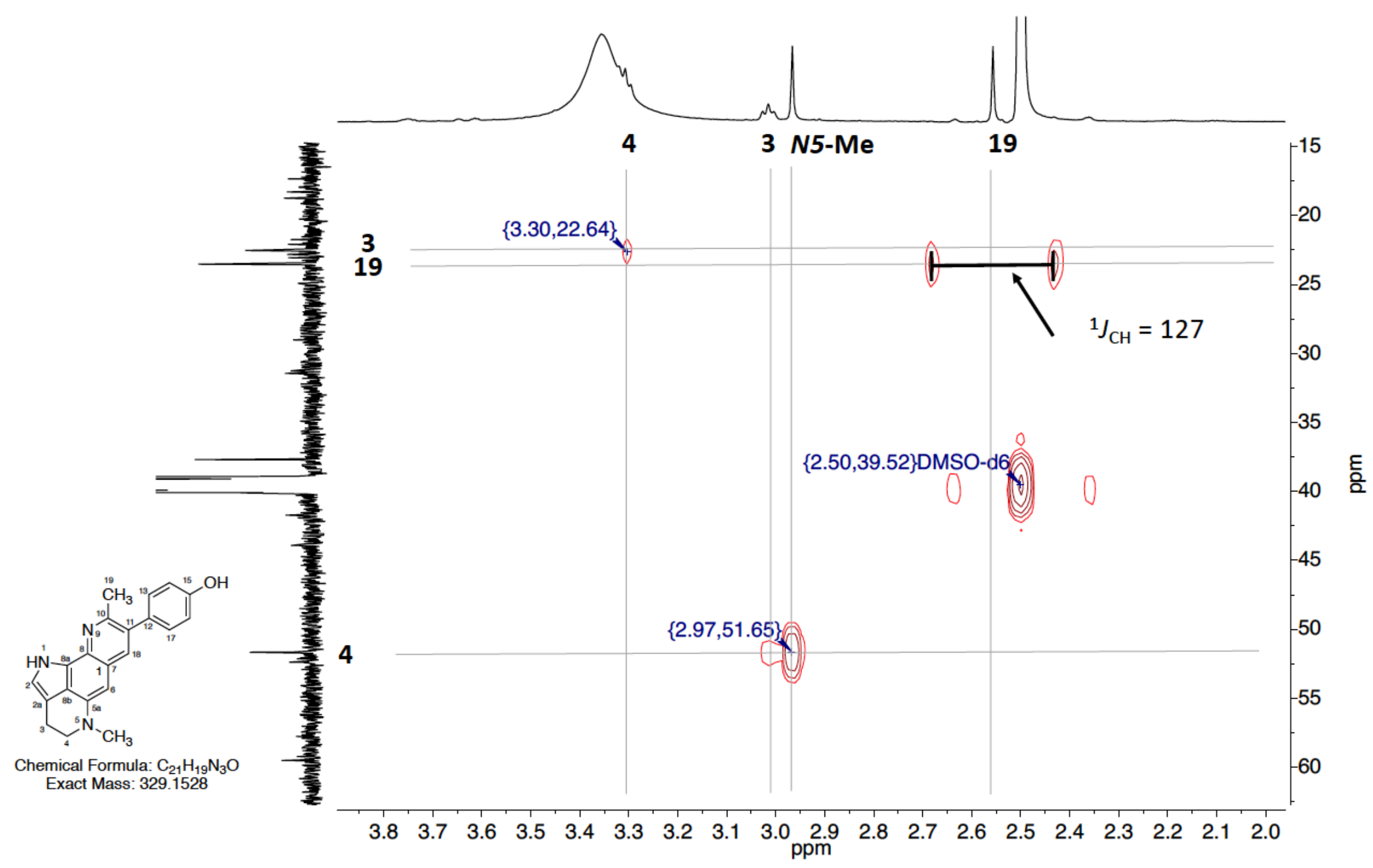

Figure S25. ${ }^{1} \mathrm{H}-{ }^{13} \mathrm{C}$ HMBC NMR Spectrum of Zyzzamine B (2) in DMSO-d6 $\left({ }^{1} \mathrm{H} 800 \mathrm{MHz},{ }^{13} \mathrm{C} 200 \mathrm{MHz}\right)$. 


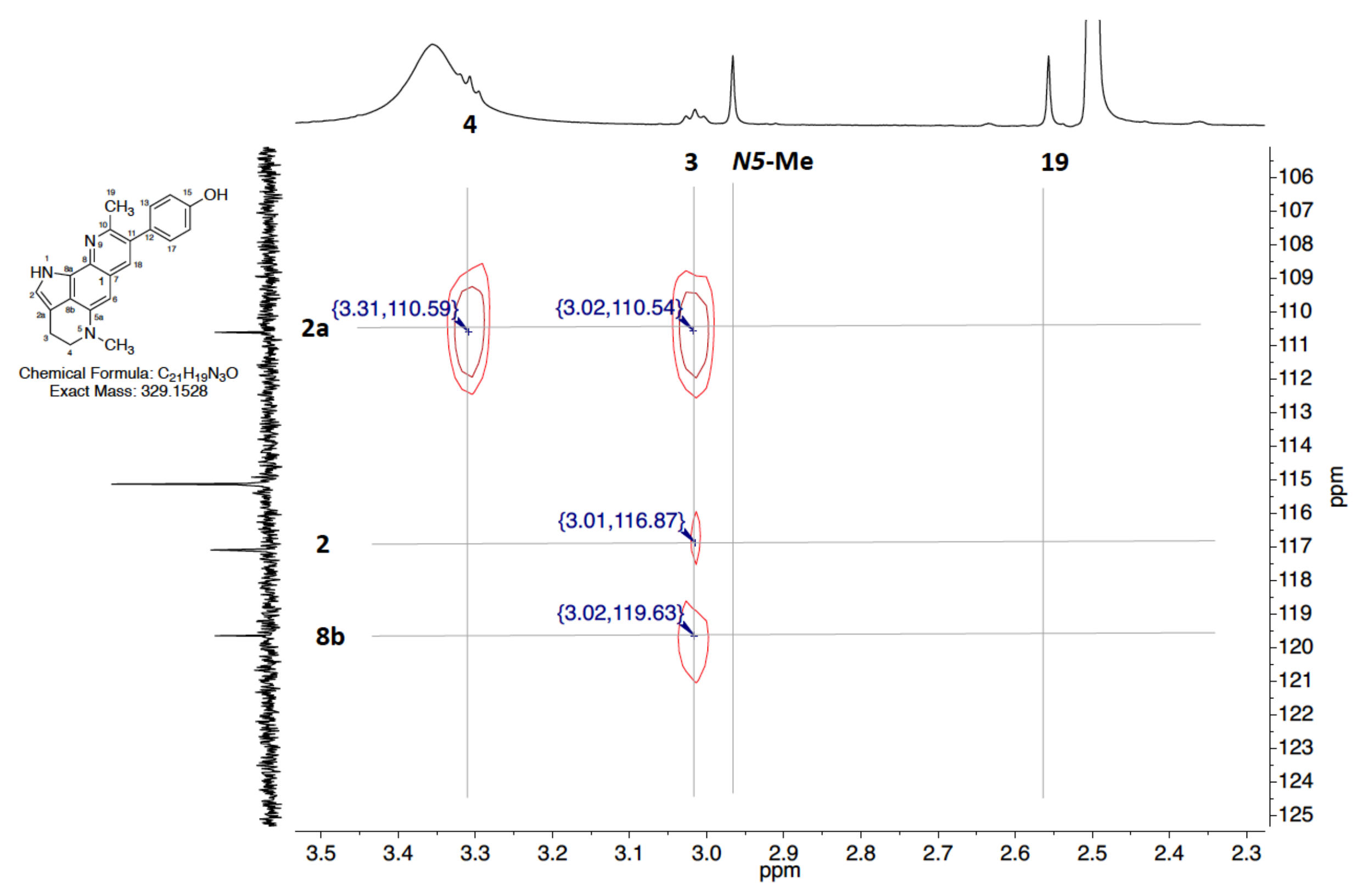

Figure S26. ${ }^{1} \mathrm{H}-{ }^{13} \mathrm{C}$ HMBC NMR Spectrum of Zyzzamine B (2) in DMSO-d $\left({ }^{1} \mathrm{H} 800 \mathrm{MHz},{ }^{13} \mathrm{C} 200 \mathrm{MHz}\right)$. 


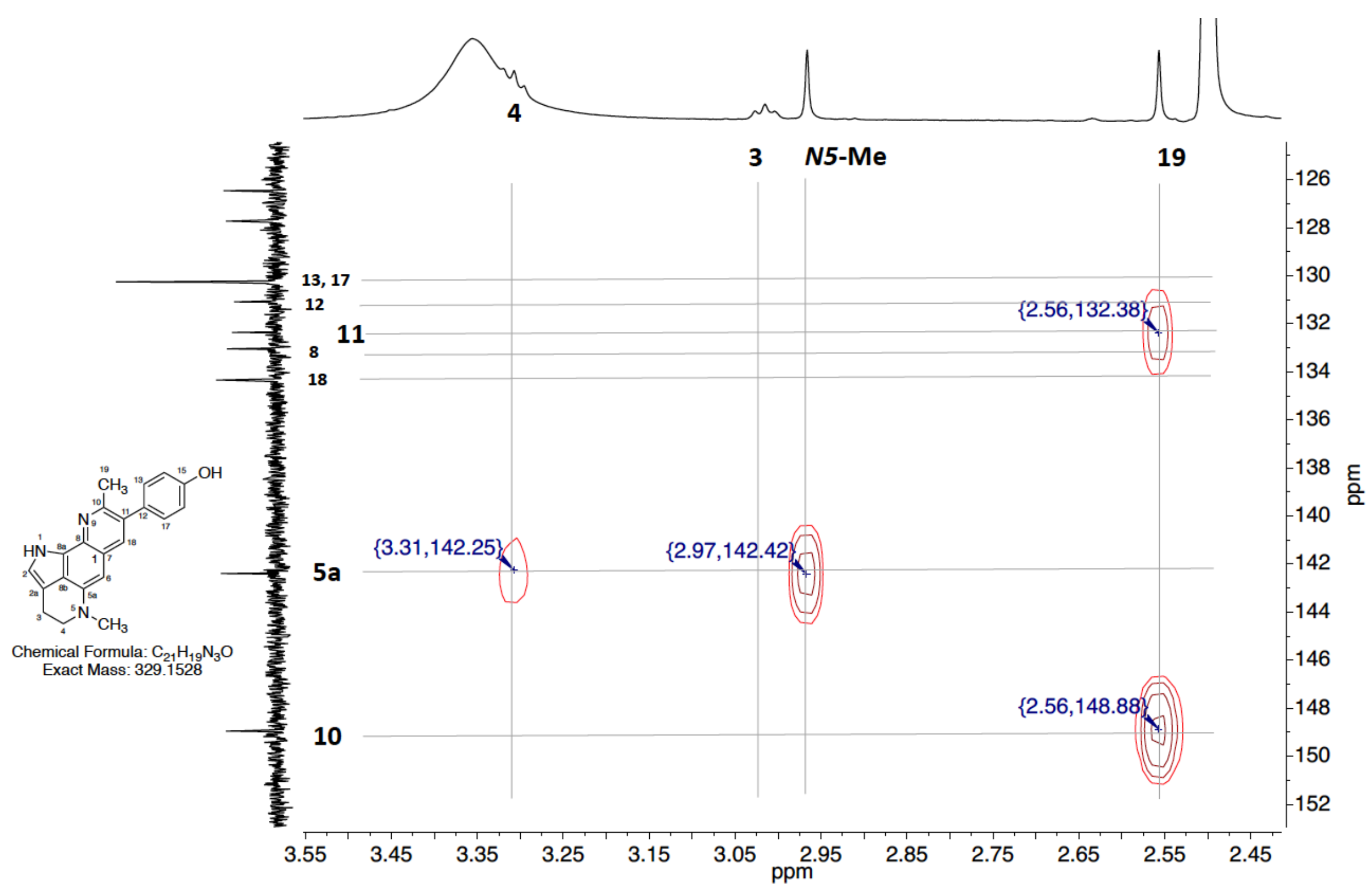

Figure S27. ${ }^{1} \mathrm{H}-{ }^{13} \mathrm{C}$ HMBC NMR Spectrum of Zyzzamine B (2) in DMSO-d6 $\left({ }^{1} \mathrm{H} 800 \mathrm{MHz},{ }^{13} \mathrm{C} 200 \mathrm{MHz}\right)$. 


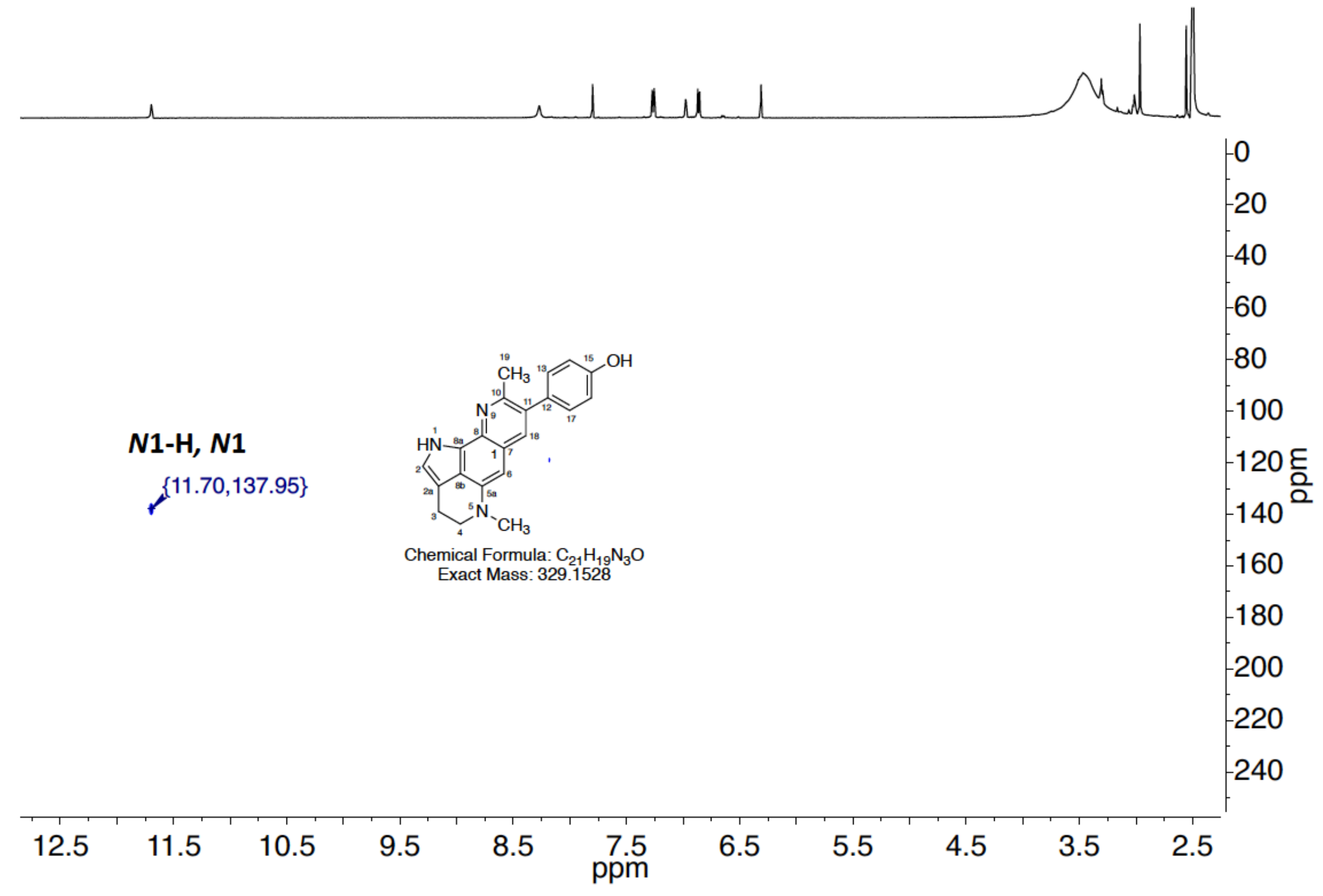

Figure S28. ${ }^{1} \mathrm{H}^{15} \mathrm{~N}$ HSQC NMR Spectrum of Zyzzamine B (2) in DMSO-d6 $\left({ }^{1} \mathrm{H} 800 \mathrm{MHz},{ }^{15} \mathrm{~N} 80 \mathrm{MHz}\right)$. 


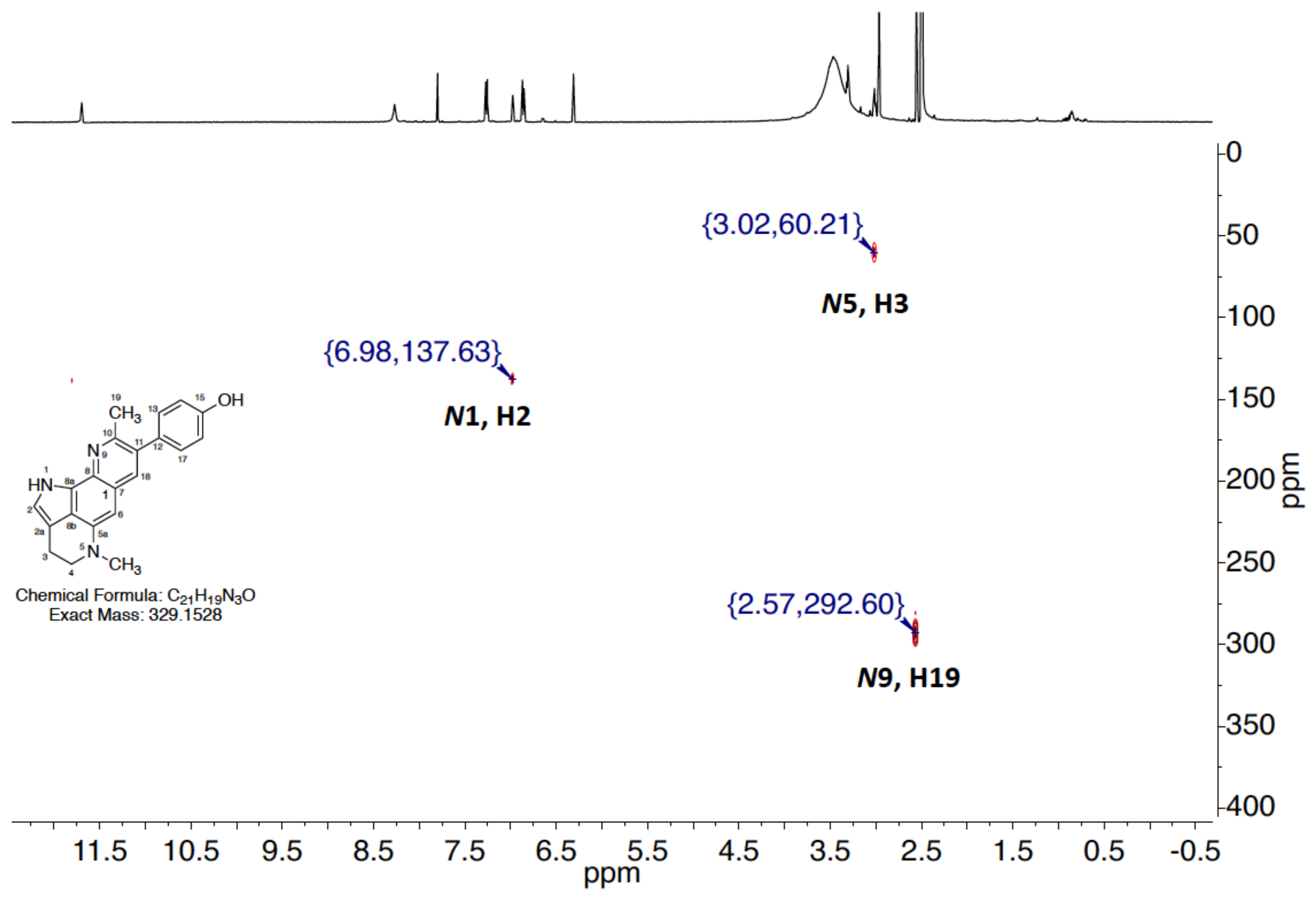

Figure S29. ${ }^{1} \mathrm{H}-{ }^{15} \mathrm{~N}$ HMBC NMR Spectrum of Zyzzamine B (2) in DMSO-d6 $\left({ }^{1} \mathrm{H} 800 \mathrm{MHz},{ }^{15} \mathrm{~N} 80 \mathrm{MHz}\right)$. 


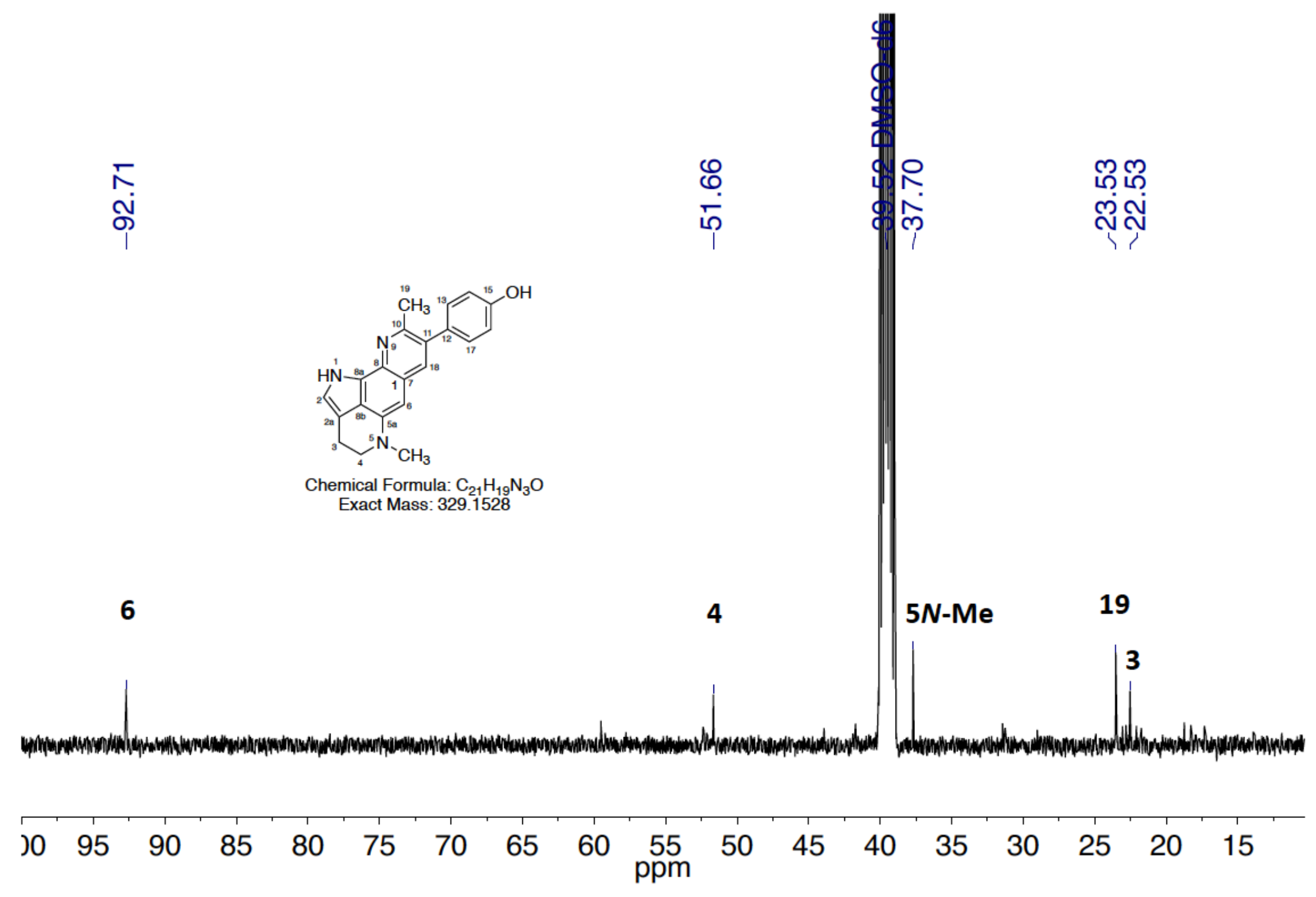

Figure S30. ${ }^{13} \mathrm{C}$ NMR Spectrum of Zyzzamine B (2) in DMSO-d6 $\left({ }^{13} \mathrm{C} 200 \mathrm{MHz}\right)$. 


\begin{tabular}{|c|c|c|c|c|c|c|c|}
\hline ப் & దீ & 웅 & 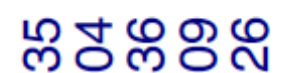 & $\mathfrak{N G}$ & ల్ & $\infty$ & $\underset{T}{N}$ \\
\hline ம் & $\stackrel{\infty}{+}$ & $\stackrel{\text { ช }}{ }$ & ர் లె లై & $\hat{N} \stackrel{\text { N }}{ }$ & के & $\stackrel{N}{\sim}$ & $\stackrel{1}{\leftarrow}$ \\
\hline$\tau$ & $\tau$ & ${ }_{1}$ & テなテテ & $\tau_{1}$ & $\Gamma_{1}$ & T & $\Gamma_{1}$ \\
\hline
\end{tabular}

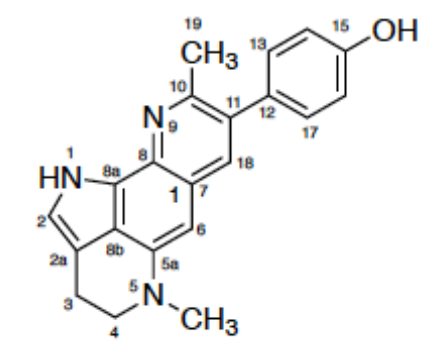

Chemical Formula: $\mathrm{C}_{21} \mathrm{H}_{19} \mathrm{~N}_{3} \mathrm{O}$ Exact Mass: 329.1528

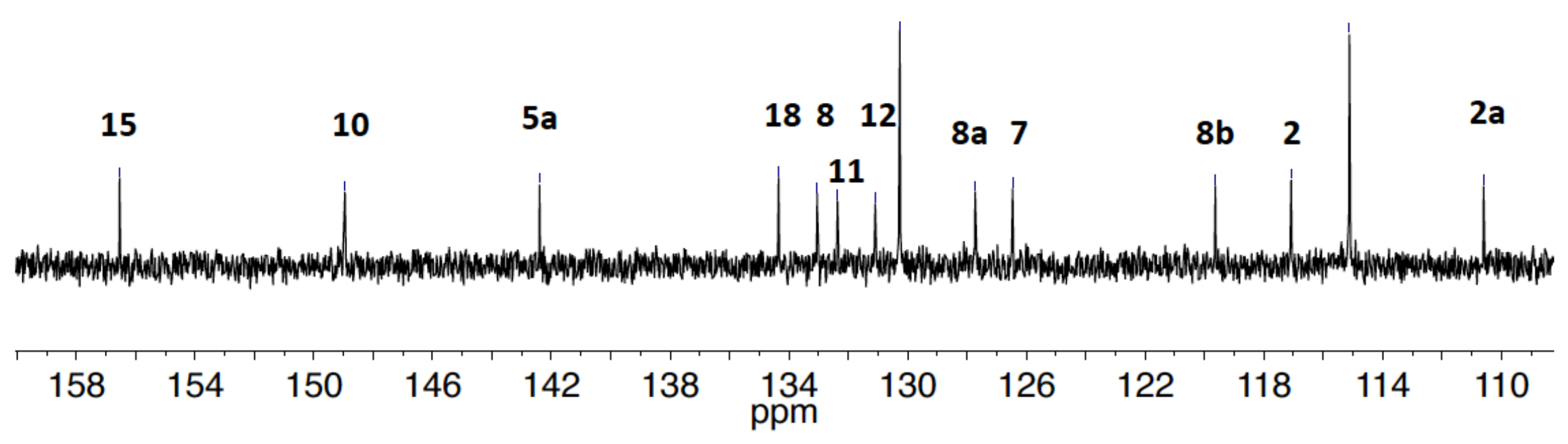

Figure S31. ${ }^{13} \mathrm{C}$ NMR Spectrum of Zyzzamine B (2) in DMSO-d6 $\left({ }^{13} \mathrm{C} 200 \mathrm{MHz}\right)$. 


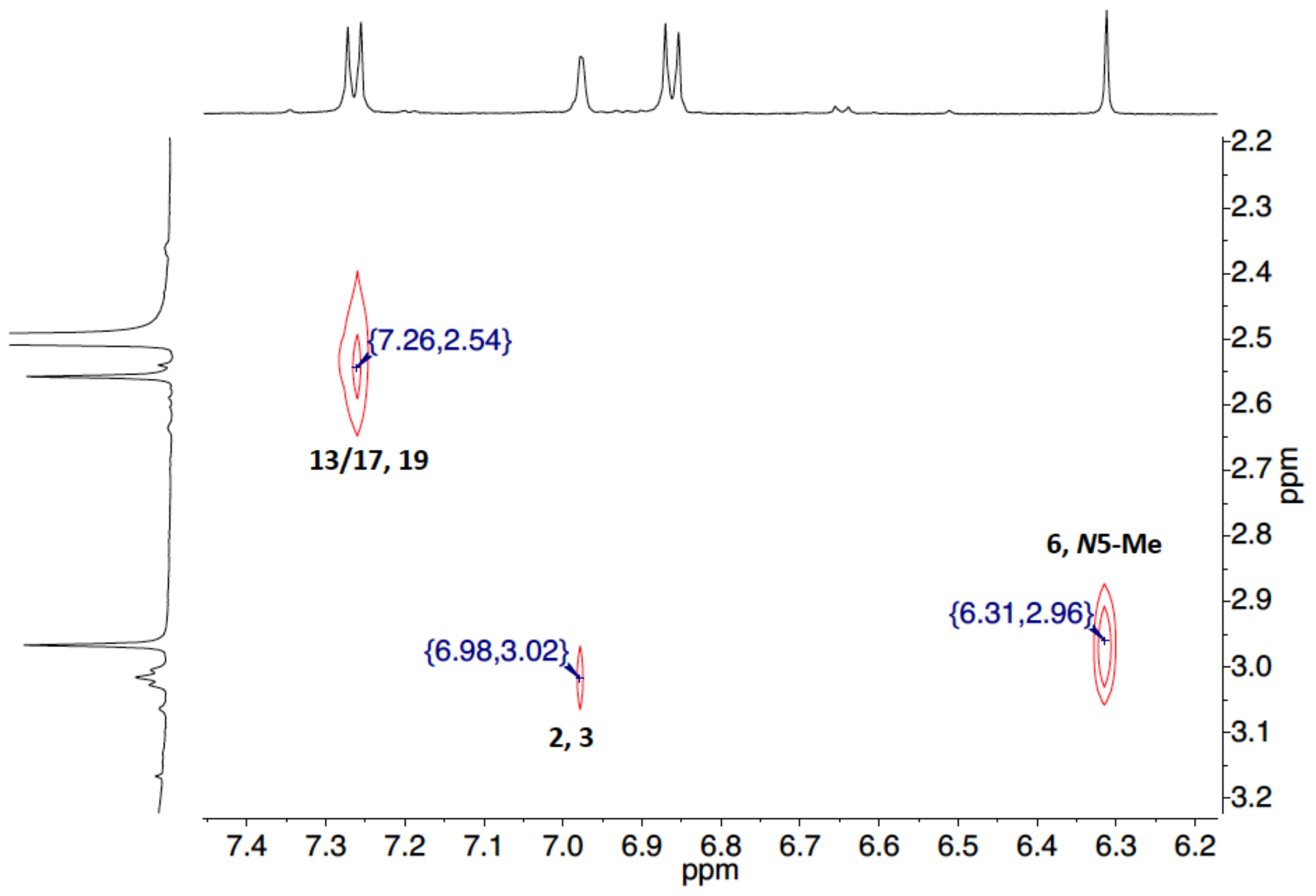

Figure S32. ${ }^{1} \mathrm{H}-{ }^{1} \mathrm{H}$ ROSEY NMR Spectrum for Zyzzamine B (2) $\left({ }^{1} \mathrm{H} 800 \mathrm{MHz}\right)$. 


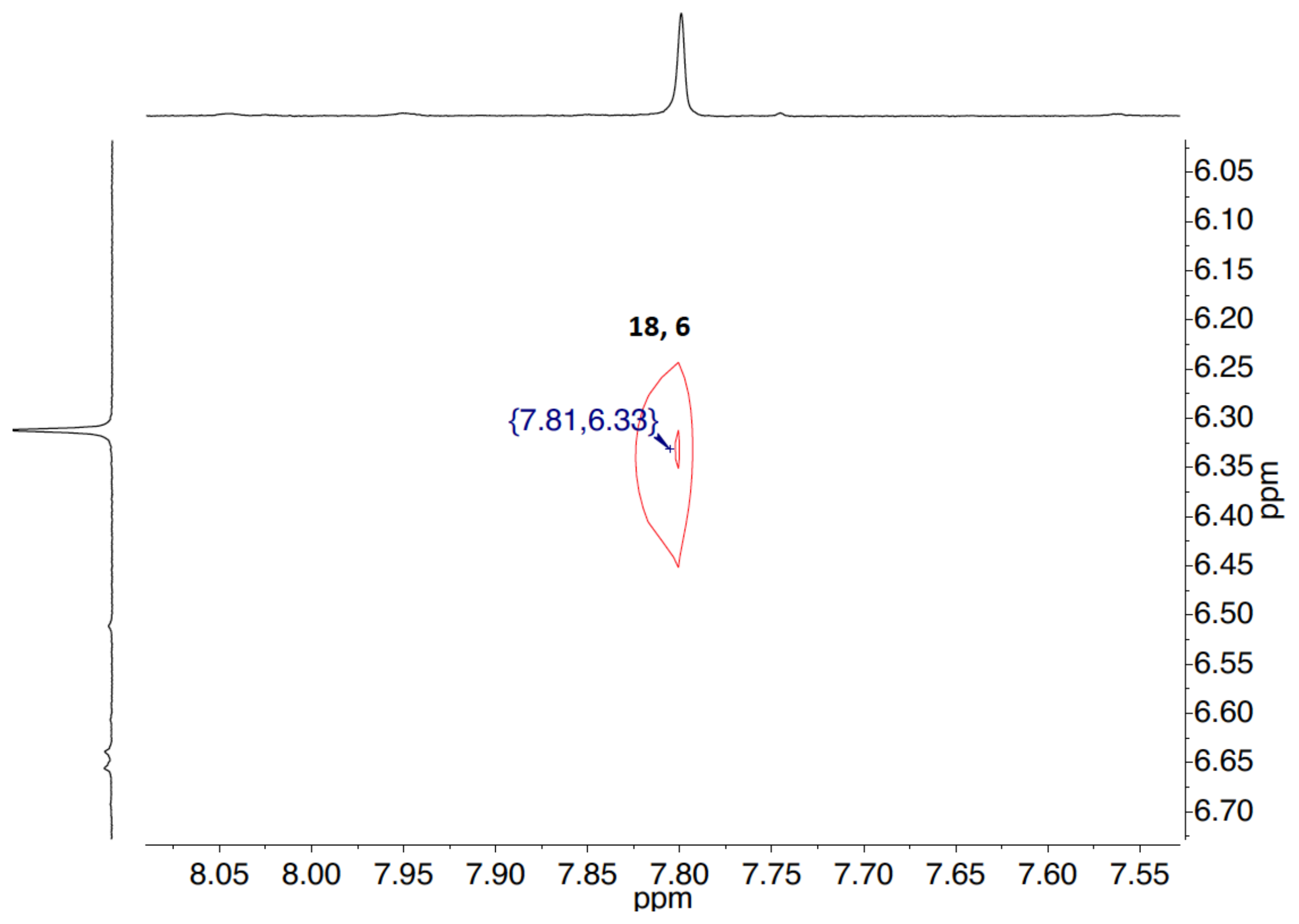

Figure S33. ${ }^{1} \mathrm{H}-{ }^{1} \mathrm{H}$ ROSEY NMR Spectrum for Zyzzamine B (2) $\left({ }^{1} \mathrm{H} 800 \mathrm{MHz}\right)$. 
Part A. Key experimental outcomes

$[1]^{1} \mathrm{H}-{ }^{1} \mathrm{H}$ ROSEY observed between $\mathrm{H} 6$ and $\mathrm{H} 18$

[2] ${ }^{1} \mathrm{H}-{ }^{1} \mathrm{H}$ ROSEY observed between $\mathrm{H} 19$ and $\mathrm{H} 13 / \mathrm{H} 17$

[3] ${ }^{1} \mathrm{H}^{-15} \mathrm{~N}$ HMBC observed from $\mathrm{H} 19$ to $N 9$

$[4]{ }^{1} \mathrm{H}-{ }^{13} \mathrm{C}$ HMBC observed from $\mathrm{H} 18$ to $\mathrm{C} 10$ \& $\mathrm{C} 11$

Part B. Structures ruled out by the above decision network data

[1] $B a, B b, B c, B d, B e, B f, B g, B h, B j, B k, B m, B n, B p, B q, B s, B t, B v, B w$ 18 unique structures ruled out

[2] Bb, Be, Bk, Bg, Bj, Bh, Bn, $\underline{B o}, \mathrm{~Bq}, \underline{B r}, \mathrm{Bt}, \mathrm{Bw}$

2 unique structures ruled out

[3] Be, Bg, Bh, Bm, Bo, Bp, Br, $\underline{B x}$

1 unique structure ruled out

[4] Bh, Bj, $\underline{B l}, \mathrm{Bm}, \mathrm{Bo}, \mathrm{Bp}, \mathrm{Br}, \underline{B i}$

2 unique structures ruled out

Part C. Final structure selected

$\boldsymbol{U}$

Part D. Summary of 24 working (candidate) structures



$\mathrm{Ba}$

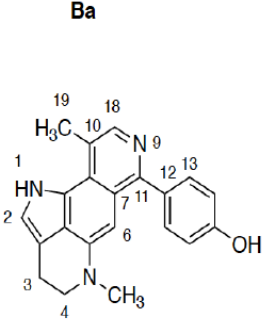

$\mathrm{Bg}$

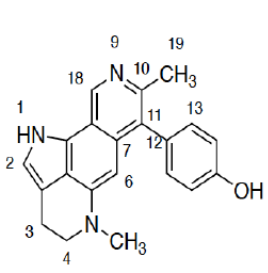

$\mathrm{Bm}$

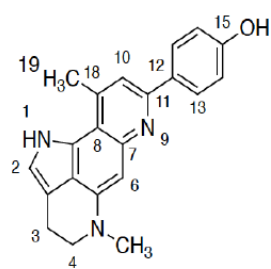

$\mathrm{Bb}$

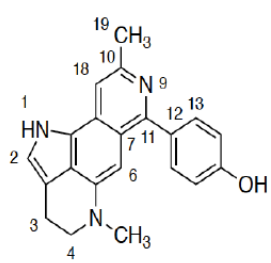

$\mathrm{Bh}$

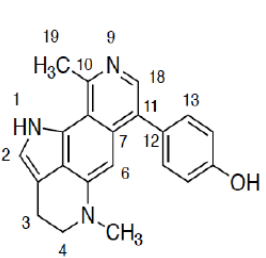

Bn
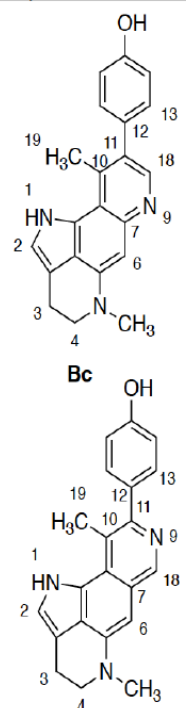

${ }^{4} \mathrm{Bi}$

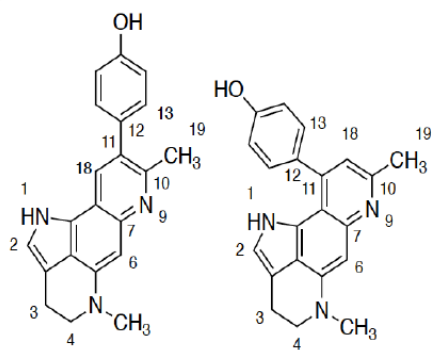

Be

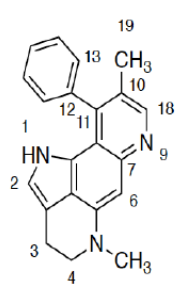

Bf

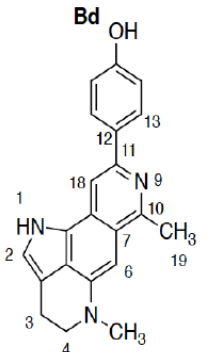

${ }^{4} \mathrm{Bj}$

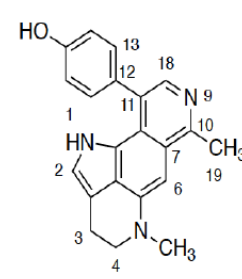

Bk

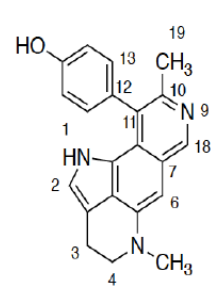

BI

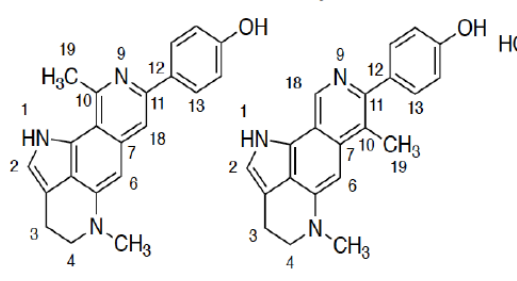

Bp

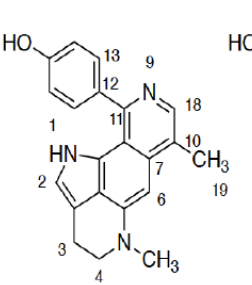

$\mathrm{Bq}$

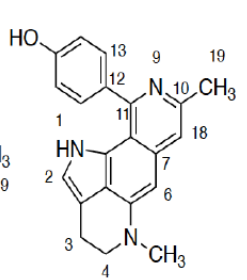

$\mathrm{Br}$

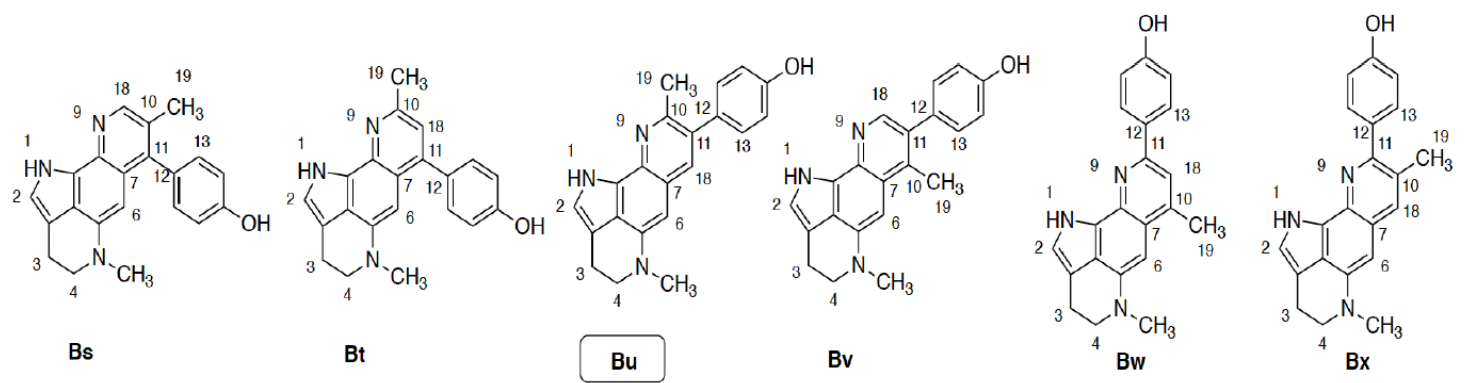

Scheme S2. Decision Network Using ${ }^{1} \mathrm{H}-{ }^{1} \mathrm{H}$ ROSEY and ${ }^{1} \mathrm{H}-{ }^{13} \mathrm{C}$ HMC to Evaluate 24 Candidate Structures for Zyzzamine B (2). 

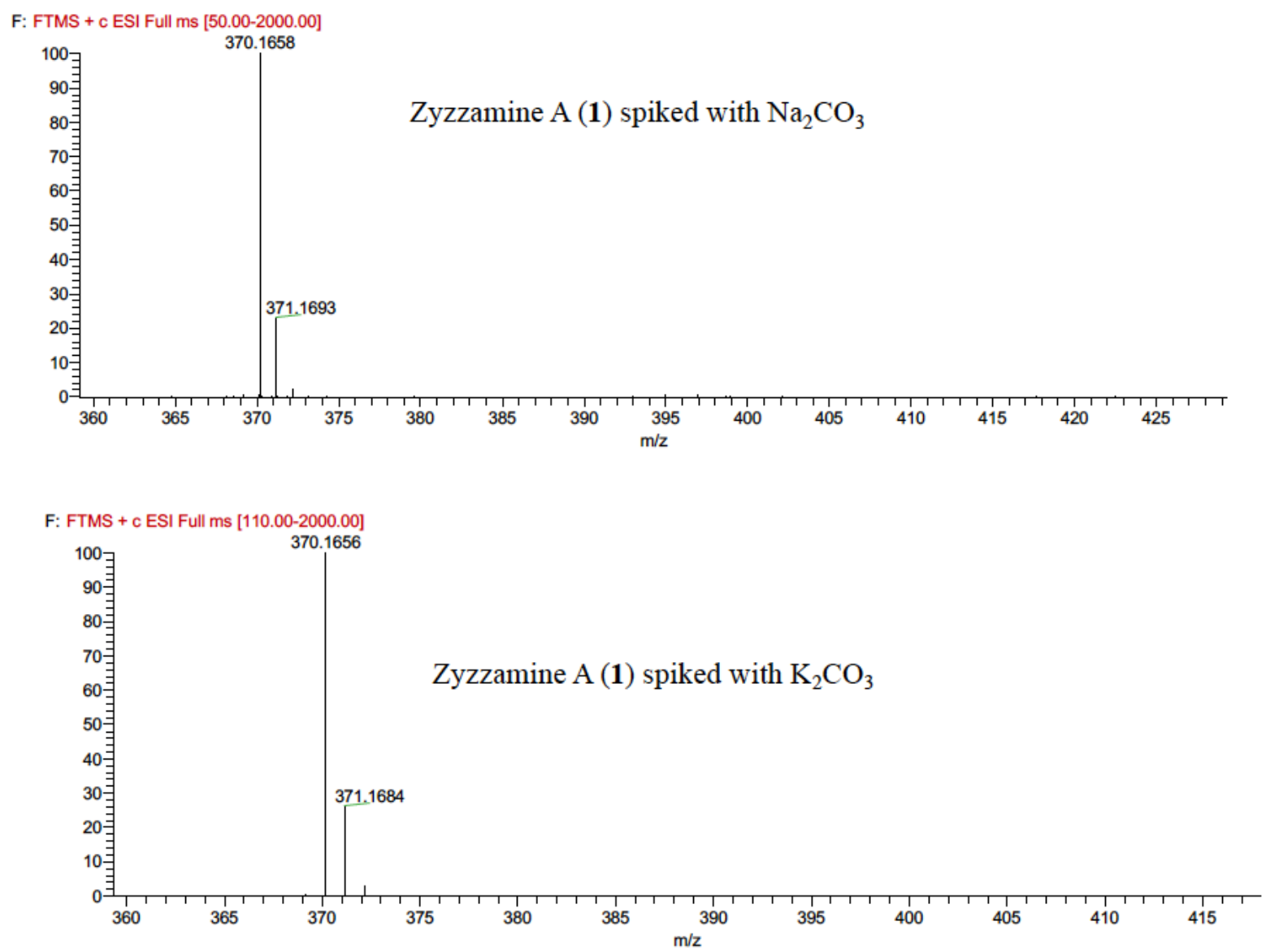

Figure S34. MS Spectra from $\mathrm{Na}^{+}$and $\mathrm{K}^{+}$Spiking Experiments for Zyzzamine A (1).

Table S1. Cell lines used in screening for Zyzzamine A (1) and B (2) cytotoxicity.

\begin{tabular}{|l|l|}
\hline rhabdomyosarcoma & SJCRH30 \\
\hline Ewing sarcoma & A-673 \\
\hline medulloblastoma & D283 \\
\hline hepatoblastoma & Hep293TT \\
\hline
\end{tabular}






Figure S35. Proposed Biosynthetic Pathway for Zyzzamine B (2). 


\begin{tabular}{|c|c|c|c|}
\hline pos. & $\begin{array}{c}\text { Experimental } \\
\boldsymbol{\delta}_{\mathbf{C}}\end{array}$ & $\begin{array}{c}\text { Aa } \\
\text { NMRDB } \\
\boldsymbol{\delta}_{\mathbf{C}}\end{array}$ & $\begin{array}{c}\text { Ab } \\
\text { NMRDB } \\
\boldsymbol{\delta}_{\mathbf{C}}\end{array}$ \\
\hline & & & \\
\hline 2 & 119.5 & 123 & 123 \\
\hline $2 \mathrm{a}$ & 111.3 & 111 & 111 \\
\hline 3 & 20.3 & 23 & 23 \\
\hline 4 & 51.3 & 50 & 49 \\
\hline $5 \mathrm{a}$ & 150.4 & 142 & 142 \\
\hline$N 5-\mathrm{Me}$ & 37.7 & 39 & 39 \\
\hline 6 & 88.3 & 124 & 99 \\
\hline 7 & 148.7 & 156 & 156 \\
\hline 8 & 123.3 & 143 & 144 \\
\hline $8 \mathrm{a}$ & 126.8 & 130 & 141 \\
\hline $8 \mathrm{~b}$ & 115.9 & 131 & 131 \\
\hline
\end{tabular}

\begin{tabular}{|c|c|c|}
\hline & \multicolumn{2}{|c|}{$\begin{array}{c}\text { Total \# of Variable } \\
\text { Shifts }\end{array}$} \\
\hline $\operatorname{diff} 3$ & three & two \\
\hline $\operatorname{diff} 4$ & none & none \\
\hline $\operatorname{diff}>5$ & five & six \\
\hline
\end{tabular}
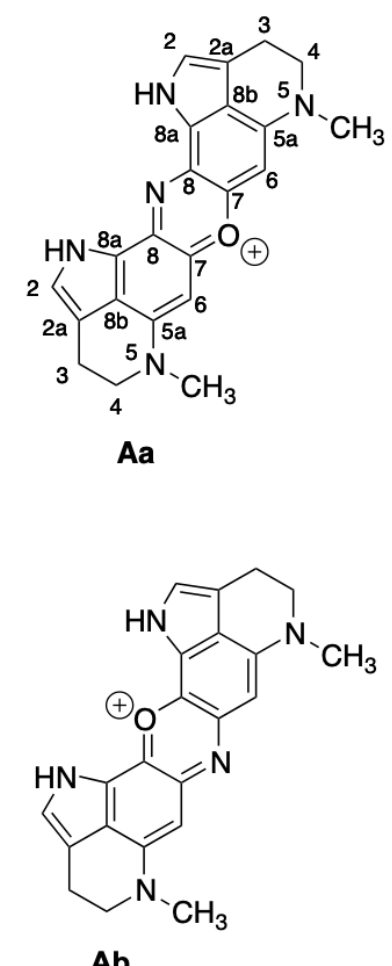

Scheme S3. Experimental vs. NMRDB calculated $\delta_{C}$ shifts for Zyzzamine A (1) structures Aa and Ab. 